\title{
In-Site and Ex-Site Date Palm Exposure to Heavy Metals Involved Infra-Individual Biomarkers Upregulation
}

\author{
Zayneb Chaâbene ${ }^{1,2, *}$, Agnieszka Rorat ${ }^{2}$, Walid Kriaa ${ }^{3}$, Imen Rekik ${ }^{4}$, Hafedh Mejdoub ${ }^{1}$, \\ Franck Vandenbulcke ${ }^{2}$ and Amine Elleuch ${ }^{1}$ \\ 1 Laboratory of Plant Biotechnology, Faculty of Sciences of Sfax, University of Sfax, Sfax 3000, Tunisia; \\ hafedh.mejdoub@fss.rnu.tn (H.M.); amineelleuch@hotmail.com (A.E.) \\ 2 Laboratoire de Génie Civil et géo-Environnement-Université de Lille 1, F-59655 Villeneuve d'Ascq, France; \\ agnieszkarorat@gmail.com (A.R.); franck.Vandenbulcke@univ-lille.fr (F.V.) \\ 3 Environmental Science Center, Qatar University, Doha P.O. Box 2713, Qatar; maswkri@yahoo.fr \\ 4 High Institute of Applied Biology of Medenine, Medenine 4119, Tunisia; imenbmc@yahoo.fr \\ * Correspondence: Zayneb.Chaabene@univ-lille.fr
}

check for updates

Citation: Chaâbene, Z.; Rorat, A.; Kriaa, W.; Rekik, I.; Mejdoub, H.; Vandenbulcke, F.; Elleuch, A. In-Site and Ex-Site Date Palm Exposure to Heavy Metals Involved Infra-Individual Biomarkers Upregulation. Plants 2021, 10, 137. https://doi.org/10.3390/ plants10010137

Received: 1 December 2020 Accepted: 6 January 2021

Published: 12 January 2021

Publisher's Note: MDPI stays neutral with regard to jurisdictional clai$\mathrm{ms}$ in published maps and institutional affiliations.

Copyright: (C) 2021 by the authors. Licensee MDPI, Basel, Switzerland. This article is an open access article distributed under the terms and conditions of the Creative Commons Attribution (CC BY) license (https:// creativecommons.org/licenses/by/ $4.0 /)$.

\begin{abstract}
As a tree of considerable importance in arid regions-date palm, Phoenix dactylifera L. survival in contaminated areas of Sfax city has drawn our attention. Leaf samples of the plants grown in the study area showed high levels of cadmium $(\mathrm{Cd})$, copper $(\mathrm{Cu})$, and chromium $(\mathrm{Cr})$. On the basis of this finding, the cellular mechanisms that explain these metal accumulations were investigated in controlled conditions. After four months of exposure to $\mathrm{Cd}, \mathrm{Cr}$, or $\mathrm{Cu}$, high bioconcentration and translocation factor ( $\mathrm{TF}>1$ ) have been shown for date palm plantlets exposed to $\mathrm{Cd}$ and low $\mathrm{TF}$ values were obtained for plantlets treated with $\mathrm{Cr}$ and $\mathrm{Cu}$. Moreover, accumulation of oxidants and antioxidant enzyme activities occurred in exposed roots to $\mathrm{Cu}$ and $\mathrm{Cd}$. Secondary metabolites, such as polyphenols and flavonoids, were enhanced in plants exposed at low metal concentrations and declined thereafter. Accumulation of flavonoids in cells may be correlated with the expression of the gene encoding Pdmate5, responsible for the transport of secondary metabolites, especially flavonoids. Other transporter genes responded positively to metal incorporation, especially Pdhma2, but also Pdabcc and Pdnramp6. The latter would be a new candidate gene sensitive to metallic stress in plants. Expressions of gene coding metal chelators were also investigated. Pdpcs1 and Pdmt 3 exhibited a strong induction in plants exposed to $\mathrm{Cr}$. These modifications of the expression of some biochemical and molecular based-markers in date palm helped to better understand the ability of the plant to tolerate metals. They could be useful in assessing heavy metal contaminations in polluted soils and may improve accumulation capacity of other plants.
\end{abstract}

Keywords: atmospheric contamination; gene expression; metallic stress; integrative biomarkers; metal accumulation

\section{Introduction}

Based on their physicochemical properties, bioactive-metals are divided into two groups: the redox-active metals, such as chromium $(\mathrm{Cr})$, copper $(\mathrm{Cu})$, and iron $(\mathrm{Fe})$, and redox-inactive metals, such as cadmium $(\mathrm{Cd})$, nickel $(\mathrm{Ni})$, aluminum (Al), and zinc $\mathrm{Zn}$ ). The metals belonging to the first group are included in a fundamental bioprocess and play a pivotal role in oxygen formation, and enzyme and protein structure [1]. They can directly generate oxidative injury via undergoing Haber-Weiss and Fenton reactions, which leads to an uncontrolled formation of reactive oxygen species (ROS) in plants. At high levels, they may cause cell homeostasis disruption, defragmentation of biological macromolecules, such as DNA (DNA strand breakage), proteins and lipids, or cell membrane and damage of photosynthetic pigments, which may trigger cell death [2]. Via inhibiting antioxidative enzymes, inducing ROS-producing enzymes (NADPH oxidases) and glutathione depletion, non-essential elements that belong to the redox-inactive metals group can indirectly inflict 
oxidative stress in living organisms [3]. Yet, in numerous cases, such as cadmium stress in liverwort Conocephalum conicum (Marchantiales; [4]), the metal induces the activity of antioxidant enzymes rather than inhibits it.

Despite its redox state, the concentration of both essential and non-essential metals is crucial for environment. They become toxic to living organisms above a critical concentration. Even those classified as essential can be toxic if present in excess [5].

A slightly elevated concentration of $\mathrm{Cu}$ can induce phytotoxicity [6], i.e., affecting plant growth and altering cellular antioxidant system [7]. The pigment and protein components of photosynthetic membranes are the targets of $\mathrm{Cu}$ ions, inducing perturbation of photosynthetic metabolisms [8]. Thereby, in presence of high $\mathrm{Cu}$ amounts, leaves suffer chlorosis and necrosis caused by the inhibition of chlorophyll and carotenoid biosynthesis, which delays the incorporation of these pigments into photosystem complexes. Cu may also reduce absorption of essential nutrients, especially Fe [9]. A non-essential metal, such as $\mathrm{Cd}$, negatively affects plant growth and development, and even causes plant death. Hazrat et al. [10] characterized Cd as an extremely worrying pollutant due to its high toxicity and large solubility in water. It is also recognized as one of the most phytotoxic heavy metal (HM) contaminants [10]. Its toxicity is correlated to the alteration of the uptake and distribution of macro- and micro-nutriments, especially Fe and Magnesium $(\mathrm{Mg})$ in plants [11]. Moreover, chlorophyll is one of its targets and chlorosis may due to $\mathrm{Fe}$ deficiency caused by root Fe(III) reductase inhibition [12], substitution of central Mg ion in the chlorophyll molecules [13], and/or by enhancement of chlorophyll catabolic activity, which may seriously affect photosynthesis [14]. Cr, the seventh most abundant metal on earth, is considered as one of the most dangerous toxic HM to living organisms and ecosystems due to its wide industrial applications [15]. Being a strong oxidizer, $\mathrm{Cr}$ (VI), the second stable form of $\mathrm{Cr}$, is highly toxic and more mobile in soil/water systems, even at low concentrations [15]. Cr phytotoxicity can result in reduction of root growth and biomass, perturbation of nutrient balance and enzyme activities, degradation of pigment status and induction of leaf chlorosis, and oxidative stress in plants [16].

Plants, especially tree species with their long reproductive cycles, have evolved a complex network of highly effective homeostatic mechanisms. They serve to maintain physiological concentrations of essential metal ions, such as $\mathrm{Cu}$, and minimize exposure to non-essential metals, such as $\mathrm{Cd}$ and $\mathrm{Cr}$ [17]. Exploring the mechanisms at infra-individual levels, using transcriptomic, proteomic, metabolomic approaches allow insight into the physiology, the biochemistry, or the cell biology of exposed organisms, and sometimes the identification of exposure biomarkers. Some mechanisms, usually described as ubiquitous, are required to minimize the damage caused by high concentrations of elements [18]. Plant cells contain well-equipped antioxidative defense elements, activated by HMs and aim to attain a new balanced redox status. This signal transduction network involves stress-related proteins, such as ROS-removing enzymes and non-enzymatic antioxidants, including low molecular mass antioxidants scavengers [3]. Other mechanisms target individual metal ions to control the accumulation, trafficking, and detoxification of metals. The latter are devoted to metals and they may occur at transcriptional level. The transcriptional expression of specific metal-responsive genes (to counteract the stress stimuli) are described as infra-individual biomarkers or molecular biomarkers [19]. When the metal comes into the plant, the plant cell activates specific genes, such as pcs [20], which control the production of chelating compounds phytochelatin (PCs; [21,22]). Metallothioneins (MTs) are able to bind metal ions and are produced from mRNA translation. The overexpression of $m t$ to increasing concentrations of HMs was observed in plants [23]. In addition, the plasma membrane transporters in plants participate in HMs stress responses, as they are involved in metal uptake and homeostasis. Numerous families of transporter genes were identified. The plant ATP-binding cassette (ABC) family plays an important role in the general detoxification mechanism. The $a b c$ expressions are enhanced by xenobiotic incorporation [24]. PCs and PC-HM complexes are transported by ABC C subfamily-type transporters [25]. P-type ATPase was reported to be involved in nutrient 
uptake and distribution [26]. Among them, the transporters belong to the heavy metal $\mathrm{P}_{1 \mathrm{~B}^{-}}$ ATPase subfamily, including HM-transporting P-type ATPase (HMA; [27]) and the Natural resistance-associated macrophage protein (NRAMP) transporters [28]. The Multidrug and toxic compound extrusion (MATE) proteins are involved in extrusion of multidrug and toxic compound from the cell.

It has been shown that date palm (Phoenix dactylifera) has great capacity to remove HMs from wastewater [29] and is able to accumulate $\mathrm{Cd}$ and $\mathrm{Cr}$ [30]. Therefore, although plant detoxification mechanisms are extensively reported in literature, little is known about date palm responses to metals stress. The species is widely cultivated in the southern part of Tunisia country, where soils around opencast mines and near the industrial sites, especially the phosphate fertilizer industry exhibit very high contents of HMs [31,32]. In fact, erosion of phosphogypsum (PG) piles, waste of the treatment of phosphate rock (PR) containing highly polluting hazardous element, can cause the contamination of the surrounding areas [33].

The aims of the present work were to (i) monitor the level of accumulation of HMs in field-exposed date palm; (ii) estimate the accumulation of several major contaminants $(\mathrm{Cd}, \mathrm{Cu}$, and $\mathrm{Cr})$ in various plant compartments in controlled conditions; (iii) explore the plant metal detoxification mechanisms involved in accumulation or avoidance of metals; and (iv) test the use of the studied infra-individual markers as biomarkers of metallic stress.

\section{Material and Methods}

\subsection{Soils and Plants Sampling from the Study Site}

The studied field was located in Sfax $\left(34^{\circ} 44^{\prime} 52.249^{\prime \prime} \mathrm{N} 10^{\circ} 45^{\prime} 58.187^{\prime \prime}\right.$ E), southern Tunisia $(237 \mathrm{~km})$. It was about $0.3 \mathrm{~km}$ far away from the PG stockpiles of the Industrial Society of Phosphoric Acid and Fertilizers (ISPAF) factory and lead melting industry (Figure 1), the main source of HMs in Sfax [34]. Beyond this distance, the land becomes occupied by industrial constructions, streets, and houses. ISPAF emitted dust was estimated annually to be around 1610 tons [33], generating two piles of PG, of more than 15 million tons [34].

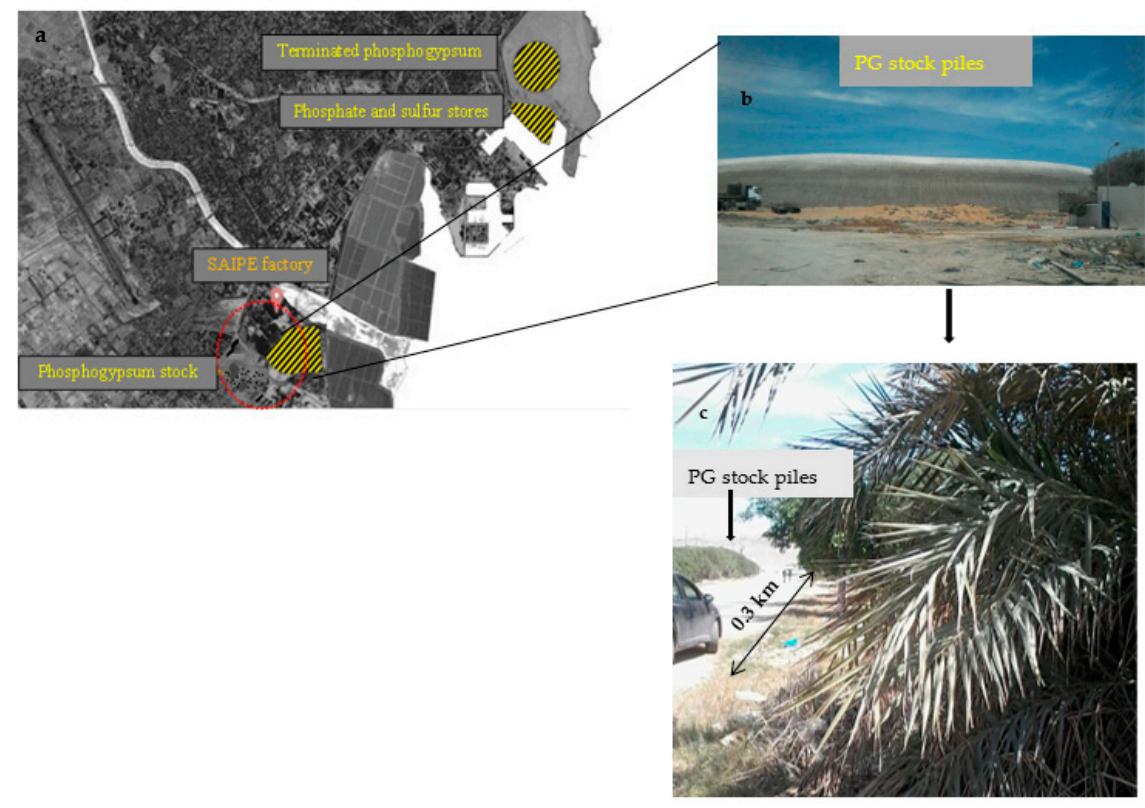

Figure 1. Show study area (a) in Sfax region $\left(34^{\circ} 44^{\prime} 52.249^{\prime \prime} \mathrm{N} 10^{\circ} 45^{\prime} 58.187^{\prime \prime} \mathrm{E}\right)$, southern Tunisia $(237 \mathrm{~km})$, were the phosphogypsum (PG) stockpiles of the Industrial Society of Phosphoric Acid and Fertilizers (ISPAF) factory (b). Soil and plant sample collections from about $0.3 \mathrm{~km}$ far away from PG stockpiles (c). 
More than 20 soil samples were collected from different locations in the distance of $0.3 \mathrm{~km}$ from the PG piles identified using the Google Maps Distance Calculator (Figure 1). The samples were collected in a depth of surface soils of all sites $(0-20 \mathrm{~cm})$. Soil samples were homogenized, collected in plastic bags, brought to laboratory, air-dried, and stored for analysis. The date palm leaves samples were collected from the same sites as the soil samples. Leaves were washed with distilled water to remove soil particles, ground with liquid nitrogen, and stored at $-20{ }^{\circ} \mathrm{C}$ for molecular analysis. Part of collected leaf samples were lyophilized and grounded in liquid nitrogen for biochemical analysis in three replicates.

\subsection{Ex-Situ Soil Contamination and Plant Transfer}

A mixture of $2 / 3$ peat and $1 / 3$ potting soil was prepared, dehydrated at $50{ }^{\circ} \mathrm{C}$, and distributed in pots; $1 \mathrm{~kg}$ per box. Amount of 10,50, and $100 \mathrm{mg}$ of $\mathrm{CdCl}_{2}$ and of 50, 100, and 500 of $\mathrm{CuSO}_{4}$ and of $\mathrm{K}_{2} \mathrm{CrO}_{4}$ powder were homogenized in distilled water. After a complete dilution, dried soils were gradually soaked with the solution by long mixing.

Seedlings of date palm of about 2 months of germination in in vitro controlled conditions produced roots between 5 and $10 \mathrm{~cm}$ lengths. They were transferred to previously prepared pots, 4 plantlets per pot. The seedlings were grown in a greenhouse at $25 \pm 3{ }^{\circ} \mathrm{C}$ and $16 \mathrm{~h}$ of photoperiod. When the plants were growing, they were sprayed with distilled water. In order to minimize leaching, the infiltrated irrigated water was recovered and reused again for irrigation. The pots were prepared in triplicates. After 4 months of growth, plants were harvested. Shoots and roots were separated and rinsed with distilled water, to ensure that outside contamination was removed. The fresh plant materials were ground in liquid nitrogen for chemical and molecular analysis. Plant tissues were oven-dried at $60{ }^{\circ} \mathrm{C}$ for $72 \mathrm{~h}$ and used for biochemical analysis. The analyses were done in triplicates.

\subsection{Soil and Plant Digestion for Metal Spectroscopic Analysis and Data Processing}

Soil and plant dried samples were lyophilized before plant material was ground to a fine powder with liquid nitrogen. One $\mathrm{mL}$ of $\mathrm{HNO}_{3}(65 \%, 108 \mathrm{~m} / \mathrm{V}$, trace pure) was added to $0.1 \mathrm{~g}$ of soil and plant tissue samples. The mixture was left at least $12 \mathrm{~h}$ at room temperature under a hood. Then, the mixture was heated to reflux for $2 \mathrm{~h}$ in a sand bath in $120{ }^{\circ} \mathrm{C}$. Before a second digestion at $180{ }^{\circ} \mathrm{C}, 1 \mathrm{~mL}$ of acid mixture $\left(\mathrm{HNO}_{3}: \mathrm{H}_{2} \mathrm{SO}_{4}: \mathrm{HClO}_{4}\right.$ : $10: 2: 3, v / v / v$ ) was added. The digests prepared in this way were then analyzed for the elements contents spectroscopically using atomic emission spectrometry with inductively coupled plasma, ICP-AES (Varian 720-ES, USA). Five elements were monitored in different compartments (soil, roots, and leaves): $\mathrm{Cd}, \mathrm{Cu}, \mathrm{Cr}, \mathrm{Mn}$, and $\mathrm{Zn}$.

The bioaccumulation factor (BAF) defined by [35] was calculated as the ratio of metal concentration in the entire plant to that in the soil [35], and is given in Equation (1).

$$
\text { BAF }=(\text { Metal }) \text { plant } /(\text { Metal }) \text { soil }
$$

The Translocation factor (TF) was described as the ratio of concentration of HM in plant shoot to that in plant root [35] and is given in Equation (2).

$$
\mathrm{TF}=(\text { Metal }) \text { shoot } /(\text { Metal }) \text { root }
$$

The Bioconcentration factor $(\mathrm{BCF})$ was calculated as ratio plant roots $\mathrm{HM}$ to that of soil [36] and is given in Equation (3).

$$
\mathrm{BCF}=(\text { Metal }) \text { root } /(\text { Metal }) \text { soil }
$$

The enrichment factor (EF) was calculated as the ratio of metal concentration in above ground plant parts over metal concentration in soil and given in Equation (4). The EF is considered ideal HM stabilizers. 
$\mathrm{EF}=((($ Metal $)$ stressed plant roots + leaves $/(\mathrm{Zn})$ stressed plant roots + leaves $) /(($ Metal $)$ doped soil $/(\mathrm{Zn})$

$$
\text { doped soil]/control) }
$$

$\mathrm{Zn}$ is used as reference element.

\subsection{Biochemical Analysis}

Hydrogen peroxide concentration was determined, as previously described by Elleuch et al. [37]. The levels of products of lipid peroxidation were measured as thiobarbituric acid reactive substances (TBARS) aldehydes, according to Rustérucci et al. [38]. The concentrations of TBARS were calculated using an extinction coefficient of $155 \mathrm{mM}^{-1} \mathrm{~cm}^{-1}$.

Total protein extraction from date palm roots and leaves was made according to Gómez-Vidal et al. [39]. Total protein content was determined spectrophotometrically according to the method of Bradford [40], using bovine serum albumin as a standard. Assays of antioxidant enzyme activities in date palm tissues were prepared for catalase (CAT), ascorbate peroxidase (APX), and superoxide dismutase (SOD) as described previously by Chaâbene et al. [41]. SOD activity was assayed using the photochemical nitro blue tetrazolium (NBT) method and measured according to Beyer and Fridovich [42]. CAT and APX activities were determined, as described by Aebi [43] and Nakano and Asada [44] methods, respectively.

The secondary metabolites were extracted by maceration of $50 \mathrm{mg}$ of tissues powder in $2 \mathrm{~mL}$ of organic solvent ( $80 \%$ acetone), under ultrasonic conditions for $45 \mathrm{~min}$, at $4{ }^{\circ} \mathrm{C}$. After centrifugation, the supernatant containing phenols was recovered. A second identical extraction was carried out to extract $30 \%$ of additional phenols to obtain a more complete dosage. The supernatants were combined before being concentrated to dryness under vacuum. The total phenols content in tissue was determined using the Folin-Ciocalteu method, described by Pinelo et al. [45]. Total flavonoids were determined according to Zhishen et al. [46]. Based on the condensation of polyphenolic compounds with vanillin in an acid medium [47], the content of tannins in roots and leaves of date palm plantlets was measured.

\subsection{Real Time qPCR Amplification}

RNA extraction and cDNA synthesis were performed, as previously described by Chaâbene et al. [25]. RNAs were isolated with the Plant RNeasy mini kit (Qiagen, Courtaboeuf, France), including the on-column DNase digestion step. Concentration and purity of the RNA samples was determined using a Spectrophotometer (SPEC-TROstar Nano Microplate Reader). Reverse transcription was performed on $1 \mathrm{mg}$ of total RNA from each sample using the random hexamer primers and the Maxima H Minus First strand (Thermo Scientific, USA) cDNA Synthesis Kit, according to the manufacturer's instructions. Primer sequences of candidate genes were designed from the conserved domain found using Primer3Plus (http:/ / frodo.wi.mit.edu/) and verified using NetPrimer and BeaconDesigner [25].

Real-time polymerase chain reaction (qPCR) amplification method, using MESA Blue qPCR Master Mix reagent kit (Eurogentec, Seraing, Belgium), was performed on reverse transcribed RNAs extracted from Phoenix dactylifera according to Brulle et al. [48]. The specific phytochelatin synthase type 1 (Pdpcs1) and metallothionein type 3 (Pdmt3), Pdabcc family, Pdhma2, Pdmate5, and Pdnramp6 specific primers were used [30]. qPCR reactions were performed with a LightCycler 480 Real Time PCR system (RocheDiagnostics, Mannheim, Germany), according to previously described procedures [43]. Real-time PCR efficiencies (E) were calculated from the given slope of the standard curve, according to equation $\mathrm{E}=10^{(-1 / \text { slope })}$. E values ranged from 1.91 to 2 (with $100 \%=2$ ) and calculated from a standard curve. The expression levels and the relative fold expression (RFE) were determined, according to previously described procedures [49]. The geometric mean of the three most stable reference genes in control and $\mathrm{Cd}, \mathrm{Cr}$, and $\mathrm{Cu}$-stressful conditions 
identified by Chaâbene et al. [30,41] was used to calculate expression of target gene levels, according to Brulle et al. [48]. Absolute quantification of genes expression levels is shown as $\log _{2}$. Pdpcs1 and Pdmt3 relative expression levels were normalized to those of the reference genes selected after gene expression validation [25].

\subsection{Statistical Analysis}

Comparative threshold values represent the mean of three repetitions of the same sample. Results were expressed as means \pm SD. All analyses were conducted using STATISTICA 10. Significant differences between parameters were tested using Tukey's HSD test after one-way and two-way ANOVA, with the type of tissues and metal concentrations as the two factors. Differences at $p<0.05$ were considered statistically significant.

\section{Results}

\subsection{Soil Contamination and Metal Concentrations in Field}

Soil collected in the contaminated area of Sfax region (Figure 1), showed Cr concentration of $28 \mathrm{mg} \mathrm{kg}^{-1}$ dry soil. Such concentration, as well as $\mathrm{Cu}$ level in field soil samples, were within permissible limits recommended by Dutch standard [50], for instance $100 \mathrm{mg} \mathrm{kg}^{-1}$ for $\mathrm{Cr}$ and $36 \mathrm{mg} \mathrm{kg}^{-1}$ for $\mathrm{Cu}$. Cd concentration was $0.9 \mathrm{mg} \mathrm{kg}^{-1}$ (Table 1) which slightly exceed Dutch standard permissible limit $\left(0.8 \mathrm{mg} \mathrm{kg}^{-1}\right)$. This was the case in the vicinity of PG stockpiles in Lebanon, where the environment was found to be contaminated with HMs attending toxic amounts considerably above-threshold of $\mathrm{Cd}, \mathrm{Zn}$, and radionuclide (U; [51]). However, $\mathrm{Cd}$ and $\mathrm{Cu}$ levels in harvested leaves of date palm in contaminated fields were almost two times higher than in the soil (Table 1). All tested metals in plant leaves exceeded the metal common range, according to the World Health Organization (WHO) [52]. Cd, Cu, and $\mathrm{Cr}$ concentrations in date palm leaves from the contaminated site of the Sfax region were $1.6,14.6$, and $17.9 \mathrm{mg} \mathrm{kg}^{-1}$, respectively. The standard limits of WHO [52] are $0.02,10$, and $1.3 \mathrm{mg} \mathrm{kg}^{-1}$ for $\mathrm{Cd}, \mathrm{Cu}$, and $\mathrm{Cr}$, respectively.

\subsection{Metal Concentrations in Plants}

Significant differences in metal concentrations were observed in various compartments (soil, roots, and leaves) compared to control plants (Table 1). After 4 months of exposure to Cd-contaminated soil, a significant bioaccumulation (i.e., BCF $>1$ ) was observed in roots of plantlets growing in 10 and $50 \mathrm{mg} \mathrm{kg}^{-1}$ of Cd-spiked soil (Table 2). Moreover, an important Cd-translocation reaction of date palm was evidenced by a TF $>1$ in samples treated with the lowest metal concentration (Table 2). It is noteworthy that $\mathrm{Cd}$ negatively influenced $\mathrm{Mn}$ uptake by date palm (Table 3). Significantly non-linear relationships between $\mathrm{Cd}$ and $\mathrm{Mn}$ was confirmed by the pairwise correlation coefficient $(r=-0.61)$, implying the existence of an antagonistic effect of $\mathrm{Cd}$ on $\mathrm{Mn}$ absorption and translocation. Similarly, $\mathrm{Cr}^{\mathrm{VI}}$ altered Mn translocation to date palm young leaves (Table 3). In addition, low TF under Cr-stress was found (Table 2). Cu concentration in roots ranges from 17.2 to $19.6 \mathrm{mg} \mathrm{kg}^{-1}$ (Table 1). BCF exceeding 1.3 and TF of 0.9 was observed for the lowest metal concentrations.

\subsection{Accumulation of Oxidants in Plant under HMs Stress}

In natural conditions, date palm plantlets generated $\mathrm{H}_{2} \mathrm{O}_{2}$ within plant cells. The accumulation of hydrogen peroxide $\left(\mathrm{H}_{2} \mathrm{O}_{2}\right)$ and the product of lipid peroxidation, in the form of TBARS significantly differ between tissues $(p<0.05)$. Leaves of the control plants accumulated oxidants more than roots (Table 4). Two-way ANOVA indicated that, except for $\mathrm{Cr}$, stress caused by high concentrations of metals $(\mathrm{Cd}$ and $\mathrm{Cu}$ ) in plant tissues influence $\mathrm{H}_{2} \mathrm{O}_{2}$ production in cells. Higher $\mathrm{Cd}$ amounts in soil further enhanced $\mathrm{H}_{2} \mathrm{O}_{2}$ and TBARS accumulation, especially in roots. However, no significant difference between roots and leaves exposed to $\mathrm{Cu}$ stress in case of $\mathrm{H}_{2} \mathrm{O}_{2}$ accumulation was shown (Table 4). The highest concentration of oxidant $\left(\mathrm{H}_{2} \mathrm{O}_{2}=709.56 \mu \mathrm{mol} / \mathrm{g}\right.$ fresh weight $(\mathrm{FW})$ was noted in roots of young plantlets of date palm grown in $100 \mathrm{mg} \mathrm{kg}^{-1}$ of $\mathrm{Cd}$ amended soils whereas, the lowest $\left(\mathrm{H}_{2} \mathrm{O}_{2}=317.79 \mu \mathrm{mol} / \mathrm{g} \mathrm{FW}\right)$ was accrued in roots of plants exposed to $100 \mathrm{mg}$ 
$\mathrm{kg}^{-1} \mathrm{Cr}$ spiked soil. In roots exposed to $500 \mathrm{mg} \mathrm{kg}^{-1}$ of $\mathrm{Cu}$ or $\mathrm{Cr}, \mathrm{H}_{2} \mathrm{O}_{2}$ amount in cells never exceeded $600 \mu \mathrm{mol} / \mathrm{g}$ FW (Table 4). Similarly, TBARS production, a biomarker of oxidative damage in cells, followed the same trend as $\mathrm{H}_{2} \mathrm{O}_{2}$ accumulation, i.e., it differed significantly between roots and leaves in Cd-stressed plants. No significant difference between tissues was shown for plants exposed to $\mathrm{Cu}$ or $\mathrm{Cr}$ treatment (Table 4).

\subsection{Enzymatic Antioxidant and Secondary Metabolites Potential in Date Palm under HMs Stress}

High levels of antioxidant enzyme activities were detected for all tested HMs (Table 4). In fact, except for date palm plantlets treated with Cr, CAT, APX, and SOD enzymes amounts were significantly much higher in leaves than in roots. CAT activity enhanced under $\mathrm{Cd}$ stress and reached its maximum at $10 \mathrm{mg} \mathrm{kg}^{-1}$ with 2.76 and $3.5 \mu \mathrm{mol}$ $\mathrm{H}_{2} \mathrm{O}_{2} / \mathrm{mg}$ protein in roots and leaves respectively. Important amounts of $\mathrm{Cu}$ and $\mathrm{Cr}$ in soils $\left(100 \mathrm{mg} \mathrm{kg}^{-1}\right)$ stimulate CAT activity more than Cd (Table 4). CAT activity continued to increase even under $500 \mathrm{mg} \mathrm{kg}^{-1}$ of $\mathrm{Cu}$ or $\mathrm{Cr}$ concentrations, while it decreased under enhancing Cd stress. APX activity showed correlation with CAT activity. It was increased when CAT activity was reduced under $50 \mathrm{mg} \mathrm{kg}^{-1} \mathrm{Cd}$ (Table 4). Indeed, APX activity was more prominent in $\mathrm{Cd}$ and $\mathrm{Cr}$ treated plantlets showing no significant differences between plants tissues treated with $\mathrm{Cr}$ (Table 4). Positive correlations were found between SOD activity and $\mathrm{Cu}$ and $\mathrm{Cr}$ concentrations in the roots and leaves of date palm young plants. The secondary plant metabolism was influenced by metal stress and plant tissues $(p<0.05)$ especially in samples treated with Cd (Table 4). Leaves accumulated more polyphenols, flavonoids, and tannins than roots. At low $\mathrm{Cd}$ stress $\left(10 \mathrm{mg} \mathrm{kg}^{-1}\right)$, flavonoids were more significantly induced compared to polyphenols and tannins (Table 4), while, no significant difference between tissues was found. Increasing $\mathrm{Cd}$ stress decreased production of nonenzymatic antioxidant metabolites. A harmful decline of tannins by more than $40 \%$ was observed in roots of plants treated with $100 \mathrm{mg} \mathrm{kg}^{-1} \mathrm{Cd}$. However, at high concentrations of $\mathrm{Cr}$, tannins content was higher than in control. Flavonoid percentage was induced in roots under cupric stress (Table 4). Contrarily, roots of plants treated with $\mathrm{Cr}$ showed no significant decline of flavonoid content, which was less influenced by this metal than by $\mathrm{Cd}$ and $\mathrm{Cu}$ (Table 4). Polyphenol content declined only in roots and leaves of plants grown in soil containing $100 \mathrm{mg} \mathrm{kg}^{-1} \mathrm{Cd}$ (Table 1). According to two-way ANOVA, polyphenol levels was influenced by the type of tissues and concentration of $\mathrm{Cd}$ and $\mathrm{Cr}$. Thereby, non-linear relationships between the two factors was shown in young date palm plant exposed to cupric stress. 
Table 1. Mean metals content in different compartments of site collected samples and ex-situ experiments.

\begin{tabular}{|c|c|c|c|c|c|c|c|c|c|c|c|c|c|c|c|c|c|c|c|}
\hline & & & & & & & & \multicolumn{12}{|c|}{ Doped Soils } \\
\hline & & \multicolumn{3}{|c|}{$\begin{array}{c}\text { Uncontaminated } \\
\text { Site }\end{array}$} & \multicolumn{3}{|c|}{ Contaminated Site } & \multicolumn{4}{|c|}{$\mathrm{Cd}\left(\mathrm{mg} \mathrm{kg}^{-1}\right)$} & \multicolumn{4}{|c|}{$\mathrm{Cu}\left(\mathrm{mg} \mathrm{kg}^{-1}\right)$} & \multicolumn{4}{|c|}{$\mathrm{Cr}\left(\mathrm{mg} \mathrm{kg}{ }^{-1}\right)$} \\
\hline & & $\mathrm{Cd}$ & $\mathrm{Cu}$ & $\mathrm{Cr}$ & $\mathrm{Cd}$ & $\mathrm{Cu}$ & $\mathrm{Cr}$ & 0 & 10 & 50 & 100 & 0 & 50 & 100 & 500 & 0 & 50 & 100 & 500 \\
\hline \multirow{2}{*}{$\begin{array}{c}\text { Metal } \\
\text { Concentration } \\
\left(\mathrm{mg} \mathrm{kg}^{-1}\right)\end{array}$} & Soil & $\begin{array}{c}0.3 \\
(0.1)\end{array}$ & $\begin{array}{c}5.4 \\
(0.4)\end{array}$ & $\begin{array}{c}0.1 \\
(0.1)\end{array}$ & $\begin{array}{c}0.9 \\
(0.1)\end{array}$ & $\begin{array}{c}7.5 \\
(0.3)\end{array}$ & $\begin{array}{l}28.0 \\
(0.8)\end{array}$ & $\begin{array}{l}0.7 \mathrm{aA} \\
(0.02)\end{array}$ & $\begin{array}{l}4.4 \mathrm{aB} \\
(0.2)\end{array}$ & $\begin{array}{c}26.3 \mathrm{aC} \\
(1.1)\end{array}$ & $\begin{array}{c}63.9 \mathrm{aD} \\
(0.9)\end{array}$ & $\begin{array}{c}5.68 \mathrm{aA} \\
(0.02)\end{array}$ & $\begin{array}{c}19.3 \mathrm{aB} \\
(1.4)\end{array}$ & $\begin{array}{c}33.4 \mathrm{aC} \\
(2.5)\end{array}$ & $\begin{array}{c}64.7 \mathrm{aD} \\
(0.9)\end{array}$ & $\begin{array}{c}0.05 \mathrm{aA} \\
(0.02)\end{array}$ & $\begin{array}{c}26.5 \mathrm{aB} \\
(2.0)\end{array}$ & $\begin{array}{c}71.8 \mathrm{aC} \\
(1.6)\end{array}$ & $\begin{array}{c}29.2 \mathrm{aD} \\
(3.9)\end{array}$ \\
\hline & Roots & - & - & - & - & - & - & $\begin{array}{l}0.2 \mathrm{bA} \\
(0.02)\end{array}$ & $\begin{array}{c}4.5 \mathrm{aAB} \\
(0.0)\end{array}$ & $\begin{array}{l}16.0 \mathrm{aBC} \\
(5.04)\end{array}$ & $\begin{array}{c}27.1 \mathrm{bC} \\
(5.1)\end{array}$ & $\begin{array}{c}4.85 \mathrm{aA} \\
(0.02)\end{array}$ & $\begin{array}{c}17.2 \mathrm{abB} \\
(2.9)\end{array}$ & $\begin{array}{c}18.8 \mathrm{bB} \\
(2.1)\end{array}$ & $\begin{array}{c}19.6 \mathrm{bB} \\
(1.9)\end{array}$ & $\begin{array}{c}0.64 \mathrm{bA} \\
(0.02)\end{array}$ & $\begin{array}{c}9.8 \mathrm{bB} \\
(0.6)\end{array}$ & $\begin{array}{c}15.4 \mathrm{bB} \\
(0.8)\end{array}$ & $\begin{array}{c}25.8 \mathrm{bC} \\
(0.2)\end{array}$ \\
\hline \multirow{3}{*}{$\begin{array}{c}\mathrm{Zn} \\
\text { Concentration } \\
\left(\mathrm{mg} \mathrm{kg}^{-1}\right)\end{array}$} & Soil & & $\begin{array}{c}9.4 \\
(0.2)\end{array}$ & & & $\begin{array}{l}15.4 \\
(0.7)\end{array}$ & & $\begin{array}{c}19.7 \mathrm{aA} \\
(0.4)\end{array}$ & $\begin{array}{c}34.9 \mathrm{aB} \\
(0.0)\end{array}$ & $\begin{array}{c}36.1 \mathrm{aC} \\
(0.1)\end{array}$ & $\begin{array}{c}41.2 \mathrm{aD} \\
(0.3)\end{array}$ & $\begin{array}{c}19.7 \mathrm{aA} \\
(0.4)\end{array}$ & $\begin{array}{c}23.91 \mathrm{aA} \\
(1.8)\end{array}$ & $\begin{array}{c}29.8 \mathrm{aA} \\
(5.3)\end{array}$ & $\begin{array}{c}32.82 \mathrm{aA} \\
(2.8)\end{array}$ & $\begin{array}{c}19.7 \mathrm{aA} \\
(0.4)\end{array}$ & $\begin{array}{c}25.1 \mathrm{aB} \\
(0.1)\end{array}$ & $\begin{array}{c}36.32 \mathrm{aC} \\
(0.2)\end{array}$ & $\begin{array}{c}29.85 \mathrm{aC} \\
(0.6)\end{array}$ \\
\hline & Roots & & - & & & - & & $\begin{array}{c}17.50 \mathrm{bA} \\
(0.6)\end{array}$ & $\begin{array}{c}10.47 \mathrm{bB} \\
(0.6)\end{array}$ & $\begin{array}{c}\text { 8.4aAB } \\
(2.0)\end{array}$ & $\begin{array}{c}\text { 6.6aAB } \\
(2.2)\end{array}$ & $\begin{array}{c}17.5 \mathrm{bA} \\
(0.6)\end{array}$ & $\begin{array}{c}16.93 \mathrm{bB} \\
(0.2)\end{array}$ & $\begin{array}{c}11.14 \mathrm{aC} \\
(0.3)\end{array}$ & $\begin{array}{c}10.2 \mathrm{aD} \\
(0.0)\end{array}$ & $\begin{array}{c}17.5 \mathrm{bA} \\
(0.6)\end{array}$ & $\begin{array}{c}14.3 \mathrm{bB} \\
(0.4)\end{array}$ & $\begin{array}{c}9.2 \mathrm{bC} \\
(3.0)\end{array}$ & $\begin{array}{c}8.8 \mathrm{bC} \\
(4.0)\end{array}$ \\
\hline & Leaves & & $\begin{array}{l}14.0 \\
(1.1)\end{array}$ & & & $\begin{array}{l}36.0 \\
(1.5)\end{array}$ & & $\begin{array}{c}29.3 \mathrm{cA} \\
(0.9)\end{array}$ & $\begin{array}{c}18.9 \mathrm{aA} \\
(1.4)\end{array}$ & $\begin{array}{c}15.5 \mathrm{bA} \\
(0.7)\end{array}$ & $\begin{array}{c}6.3 \mathrm{bB} \\
(0.5)\end{array}$ & $\begin{array}{c}29.3 \mathrm{cA} \\
(0.9)\end{array}$ & $\begin{array}{c}22.8 \mathrm{abB} \\
(2.8)\end{array}$ & $\begin{array}{c}10.87 \mathrm{aC} \\
(0.2)\end{array}$ & $\begin{array}{c}8.85 \mathrm{aC} \\
(0.0)\end{array}$ & $\begin{array}{c}29.3 \mathrm{cA} \\
(0.9)\end{array}$ & $\begin{array}{c}18.4 \mathrm{bB} \\
(0.3)\end{array}$ & $\begin{array}{c}11.81 \mathrm{aC} \\
(4.0)\end{array}$ & $\begin{array}{c}10.82 \mathrm{aC} \\
(0.1)\end{array}$ \\
\hline
\end{tabular}

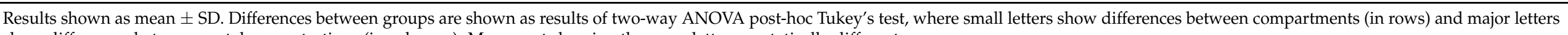
show differences between metal concentrations (in columns). Means not showing the same letter are statically different.

Table 2. Values of related metal accumulation factors. Results shown as mean $\pm \mathrm{SD}$.

\begin{tabular}{|c|c|c|c|c|c|c|c|c|c|c|c|c|}
\hline & \multicolumn{4}{|c|}{$\mathrm{Cd}\left(\mathrm{mg} \mathrm{kg}^{-1}\right)$} & \multicolumn{4}{|c|}{$\mathrm{Cu}\left(\mathrm{mg} \mathrm{kg} \mathrm{kg}^{-1}\right)$} & \multicolumn{4}{|c|}{$\mathrm{Cr}\left(\mathrm{mg} \mathrm{kg}^{-1}\right)$} \\
\hline & 0 & 10 & 50 & 100 & 0 & 50 & 100 & 500 & 0 & 50 & 100 & 500 \\
\hline BAF & $0.64(0.0)$ & $0.74(0.2)$ & $0.46(0.1)$ & $0.24(0.1)$ & $0.29(0.0)$ & $0.49(0.0)$ & $0.38(0.1)$ & $0.21(0.1)$ & $0.38(0.0)$ & $0.27(0.0)$ & $0.12(0.0)$ & $0.04(0.0)$ \\
\hline EF & $0.15(0.4)$ & $4.94(0.4)$ & $4.48(0.3)$ & $5.87(0.2)$ & $0.64(0.0)$ & $1.28(0.5)$ & $1.99(0.1)$ & $1.39(0.7)$ & $5.55(0.4)$ & $0.08(0.0)$ & $0.1(0.02)$ & $0.3(0.0)$ \\
\hline $\mathrm{TF}$ & $0.23(0)$. & $1.02(0.0)$ & $0.60(0.0)$ & $0.42(0.1)$ & $1.77(0)$. & $0.9(0.2)$ & $0.56(0.0)$ & $0.30(0.0)$ & $1.5(0.0)$ & $0.90(0.1)$ & $0.56(0.0)$ & $0.30(0.0)$ \\
\hline BCF & $0.93(0.1)$ & $1.72(0.0)$ & $1.07(0.0)$ & $0.66(0.1)$ & $0.19(0.1)$ & $1.37(0.1)$ & $0.98(0.3)$ & $0.51(0.0)$ & $1.89(0.1)$ & $0.37(0.0)$ & $0.21(0.0)$ & $0.08(0.0)$ \\
\hline
\end{tabular}


Table 3. Mn content in different compartments of site collected samples and ex-situ experiments.

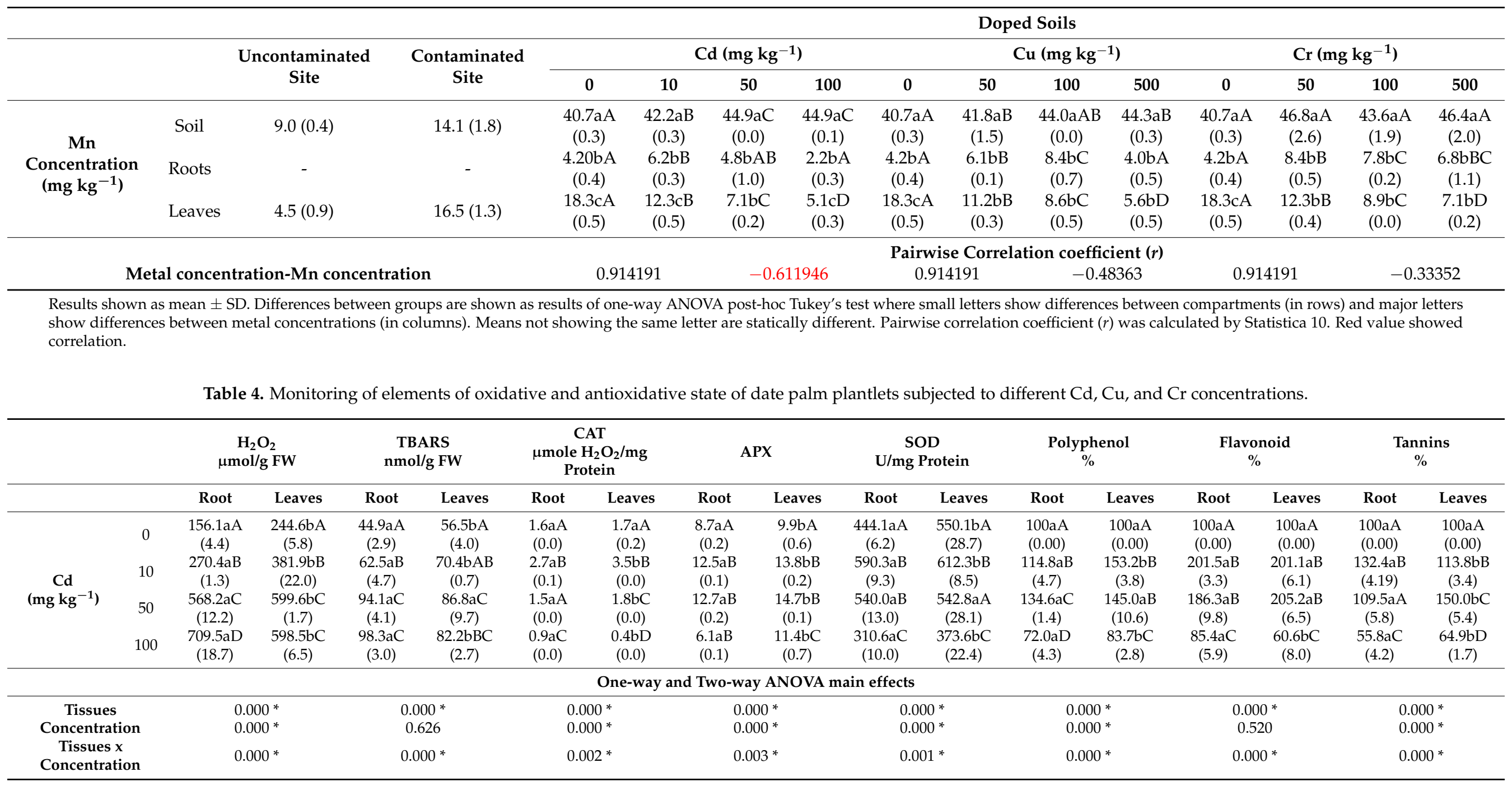


Table 4. Cont.

\begin{tabular}{|c|c|c|c|c|c|c|c|c|c|c|c|c|c|c|c|c|c|}
\hline & & \multicolumn{2}{|c|}{$\begin{array}{c}\mathrm{H}_{2} \mathrm{O}_{2} \\
\mu \mathrm{mol} / \mathrm{g} \mathrm{FW}\end{array}$} & \multicolumn{2}{|c|}{$\begin{array}{c}\text { TBARS } \\
\text { nmol/g FW }\end{array}$} & \multicolumn{2}{|c|}{$\begin{array}{c}\mathrm{CAT} \\
\mu \text { mole } \mathrm{H}_{2} \mathrm{O}_{2} / \mathrm{mg} \\
\text { Protein }\end{array}$} & \multicolumn{2}{|c|}{ APX } & \multicolumn{2}{|c|}{$\begin{array}{c}\text { SOD } \\
\text { U/mg Protein }\end{array}$} & \multicolumn{2}{|c|}{$\begin{array}{c}\text { Polyphenol } \\
\%\end{array}$} & \multicolumn{2}{|c|}{$\begin{array}{c}\text { Flavonoid } \\
\quad \%\end{array}$} & \multicolumn{2}{|c|}{$\begin{array}{c}\text { Tannins } \\
\%\end{array}$} \\
\hline & & Root & Leaves & Root & Leaves & Root & Leaves & Root & Leaves & Root & Leaves & Root & Leaves & Root & Leaves & Root & Leaves \\
\hline \multirow{2}{*}{$\begin{array}{c}\mathrm{Cu} \\
\left(\mathrm{mg} \mathrm{kg}^{-1}\right)\end{array}$} & 50 & $\begin{array}{c}202.9 \mathrm{aB} \\
(4.6)\end{array}$ & $\begin{array}{c}248.4 \mathrm{aA} \\
(20.9)\end{array}$ & $\begin{array}{l}44.3 \mathrm{aA} \\
(3.4)\end{array}$ & $\begin{array}{c}\text { 63.5bAB } \\
(3.1)\end{array}$ & $\begin{array}{c}2.0 \mathrm{aB} \\
(0.1)\end{array}$ & $\begin{array}{c}2.0 \mathrm{aAB} \\
(0.1)\end{array}$ & $\begin{array}{c}10.9 \mathrm{aB} \\
(0.1)\end{array}$ & $\begin{array}{c}11.8 \mathrm{bB} \\
(0.2)\end{array}$ & $\begin{array}{c}583.8 \mathrm{aB} \\
(13.8)\end{array}$ & $\begin{array}{c}633.5 \mathrm{bB} \\
(17.7)\end{array}$ & $\begin{array}{c}116.7 \mathrm{aA} \\
(7.0)\end{array}$ & $\begin{array}{c}127.2 \mathrm{aB} \\
(3.8)\end{array}$ & $\begin{array}{c}238.1 \mathrm{aA} \\
(6.6)\end{array}$ & $\begin{array}{c}247.8 \mathrm{aB} \\
(1)\end{array}$ & $\begin{array}{l}148.4 \mathrm{aB} \\
(7)\end{array}$ & $\begin{array}{l}151.8 \mathrm{aB} \\
(6)\end{array}$ \\
\hline & 100 & $\begin{array}{c}319.4 \mathrm{aC} \\
(17.8)\end{array}$ & $\begin{array}{c}325.0 \mathrm{aB} \\
(25.0)\end{array}$ & $\begin{array}{c}53.5 \mathrm{aA} \\
(2.31)\end{array}$ & $\begin{array}{c}69.2 \mathrm{bBC} \\
(3.17)\end{array}$ & $\begin{array}{c}2.4 \mathrm{aC} \\
(0.1)\end{array}$ & $\begin{array}{c}2.4 \mathrm{bBC} \\
(0.0)\end{array}$ & $\begin{array}{c}12.5 \mathrm{aBC} \\
(0.1)\end{array}$ & $\begin{array}{c}13.7 \mathrm{bC} \\
(0.2)\end{array}$ & $\begin{array}{c}660.8 \mathrm{aC} \\
(12.3)\end{array}$ & $\begin{array}{c}717.1 \mathrm{bC} \\
(9.5)\end{array}$ & $\begin{array}{c}151.5 \mathrm{aB} \\
(6.3)\end{array}$ & $\begin{array}{c}185.2 \mathrm{bC} \\
(12.5)\end{array}$ & $\begin{array}{c}271.2 \mathrm{aA} \\
(18.1)\end{array}$ & $\begin{array}{c}184.8 \mathrm{bC} \\
(11.4)\end{array}$ & $\begin{array}{c}162.6 \mathrm{aC} \\
(6.5)\end{array}$ & $\begin{array}{c}179.8 \mathrm{bC} \\
(2.8)\end{array}$ \\
\hline \multicolumn{18}{|c|}{ One-way and Two-way ANOVA main effects } \\
\hline \multirow{3}{*}{\multicolumn{2}{|c|}{$\begin{array}{c}\text { Tissues } \\
\text { Concentration } \\
\text { Tissues } x \\
\text { Concentration }\end{array}$}} & \multirow{2}{*}{\multicolumn{2}{|c|}{$\begin{array}{l}0.000 \text { * } \\
0.015 \text { * }\end{array}$}} & \multirow{2}{*}{\multicolumn{2}{|c|}{$\begin{array}{l}0.000 \text { * } \\
0.000 \text { * }\end{array}$}} & \multirow{2}{*}{\multicolumn{2}{|c|}{$\begin{array}{l}0.000 \text { * } \\
0.340 \text { * }\end{array}$}} & \multirow{2}{*}{\multicolumn{2}{|c|}{$\begin{array}{l}0.000 \text { * } \\
0.001 \text { * }\end{array}$}} & \multirow{2}{*}{\multicolumn{2}{|c|}{$\begin{array}{l}0.000 * \\
0.000 *\end{array}$}} & \multirow{2}{*}{\multicolumn{2}{|c|}{$\begin{array}{c}0.000 * \\
0.990\end{array}$}} & \multirow{2}{*}{\multicolumn{2}{|c|}{$\begin{array}{l}0.613 \\
0.539\end{array}$}} & \multicolumn{2}{|c|}{$0.000 *$} \\
\hline & & & & & & & & & & & & & & & & & \\
\hline & & \multicolumn{2}{|c|}{0.352} & \multicolumn{2}{|c|}{0.175} & \multicolumn{2}{|c|}{0.780} & \multicolumn{2}{|c|}{0.453} & \multicolumn{2}{|c|}{0.350} & \multicolumn{2}{|c|}{0.363} & \multicolumn{2}{|c|}{0.648} & \multicolumn{2}{|c|}{0.034 * } \\
\hline \multirow{4}{*}{$\begin{array}{c}\mathrm{Cr} \\
\left(\mathrm{mg} \mathrm{kg}^{-1}\right)\end{array}$} & 0 & $\begin{array}{c}\text { 156.1aA } \\
(4.4)\end{array}$ & $\begin{array}{c}244.6 \mathrm{bA} \\
(5.8)\end{array}$ & $\begin{array}{c}44.9 \mathrm{aA} \\
(2.9)\end{array}$ & $\begin{array}{c}56.5 \mathrm{bA} \\
(4.0)\end{array}$ & $\begin{array}{c}1.6 \mathrm{aA} \\
(0.0)\end{array}$ & $\begin{array}{c}1.7 \mathrm{aA} \\
(0.2)\end{array}$ & $\begin{array}{c}8.7 \mathrm{aA} \\
(0.2)\end{array}$ & $\begin{array}{c}9.9 \mathrm{bA} \\
(0.6)\end{array}$ & $\begin{array}{c}444.1 \mathrm{aA} \\
(6.2)\end{array}$ & $\begin{array}{c}550.1 \mathrm{bA} \\
(28.7)\end{array}$ & $\begin{array}{l}100 \mathrm{aA} \\
(0.00)\end{array}$ & $\begin{array}{l}100 \mathrm{aA} \\
(0.00)\end{array}$ & $\begin{array}{l}100 \mathrm{aA} \\
(0.00)\end{array}$ & $\begin{array}{c}100 \mathrm{aA} \\
(0.00)\end{array}$ & $\begin{array}{c}100 \mathrm{aA} \\
(0.00)\end{array}$ & $\begin{array}{l}100 \mathrm{aA} \\
(0.00)\end{array}$ \\
\hline & 50 & $\begin{array}{c}183.9 \mathrm{aA} \\
(18.1)\end{array}$ & $\begin{array}{c}268.5 \mathrm{bA} \\
(16.0)\end{array}$ & $\begin{array}{l}45.1 \mathrm{aA} \\
(1.9)\end{array}$ & $\begin{array}{l}59.0 \mathrm{bA} \\
(1.9)\end{array}$ & $\begin{array}{c}2.1 \mathrm{aAB} \\
(0.0)\end{array}$ & $\begin{array}{c}1.8 \mathrm{aAB} \\
(0.1)\end{array}$ & $\begin{array}{c}11.0 \mathrm{aB} \\
(0.1)\end{array}$ & $\begin{array}{c}11.4 \mathrm{bB} \\
(0.1)\end{array}$ & $\begin{array}{c}503.5 \mathrm{aB} \\
(7.6)\end{array}$ & $\begin{array}{c}576.0 \mathrm{bA} \\
(9.7)\end{array}$ & $\begin{array}{c}120.2 \mathrm{aB} \\
(1.2)\end{array}$ & $\begin{array}{c}152.6 \mathrm{bB} \\
(2.6)\end{array}$ & $\begin{array}{c}152.5 \mathrm{aB} \\
(3.6)\end{array}$ & $\begin{array}{c}130.7 \mathrm{bB} \\
(1.3)\end{array}$ & $\begin{array}{c}169.6 \mathrm{aB} \\
(3.5)\end{array}$ & $\begin{array}{c}153.0 \mathrm{bB} \\
(5.9)\end{array}$ \\
\hline & 100 & $\begin{array}{c}317.7 \mathrm{aB} \\
(3.2)\end{array}$ & $\begin{array}{c}347.9 \mathrm{aB} \\
(32.7)\end{array}$ & $\begin{array}{c}60.6 \mathrm{aBB} \\
(7.5)\end{array}$ & $\begin{array}{c}72.6 \mathrm{aB} \\
(2.4)\end{array}$ & $\begin{array}{c}2.4 \mathrm{aBC} \\
(0.3)\end{array}$ & $\begin{array}{c}2.2 \mathrm{aBC} \\
(0.1)\end{array}$ & $\begin{array}{c}12.6 \mathrm{aC} \\
(0.3)\end{array}$ & $\begin{array}{c}12.2 \mathrm{aB} \\
(0.2)\end{array}$ & $\begin{array}{c}617.1 \mathrm{aC} \\
(26.8)\end{array}$ & $\begin{array}{c}684.5 \mathrm{bB} \\
(11.1)\end{array}$ & $\begin{array}{c}160.6 \mathrm{aC} \\
(6.7)\end{array}$ & $\begin{array}{c}217.8 \mathrm{bC} \\
(0.4)\end{array}$ & $\begin{array}{c}272.8 \mathrm{aC} \\
(8.3)\end{array}$ & $\begin{array}{c}210.2 \mathrm{bC} \\
(8.9)\end{array}$ & $\begin{array}{c}127.0 \mathrm{aC} \\
(3.5)\end{array}$ & $\begin{array}{c}181.3 \mathrm{bC} \\
(6.2)\end{array}$ \\
\hline & 500 & $\begin{array}{c}589.4 \mathrm{aC} \\
(11.7)\end{array}$ & $\begin{array}{c}520.9 \mathrm{bC} \\
(27.6)\end{array}$ & $\begin{array}{c}83.0 \mathrm{aC} \\
(2.7)\end{array}$ & $\begin{array}{c}87.1 \mathrm{aC} \\
(0.8)\end{array}$ & $\begin{array}{c}2.7 \mathrm{aC} \\
(0.2)\end{array}$ & $\begin{array}{c}2.3 \mathrm{aC} \\
(0.1)\end{array}$ & $\begin{array}{c}14.5 \mathrm{aD} \\
(0.4)\end{array}$ & $\begin{array}{c}14.5 \mathrm{aC} \\
(0.3)\end{array}$ & $\begin{array}{c}\text { 730.7aD } \\
(11.6)\end{array}$ & $\begin{array}{c}787 \mathrm{bC} \\
(16.6)\end{array}$ & $\begin{array}{c}\text { 130.1aD } \\
(1.0)\end{array}$ & $\begin{array}{c}113.6 \mathrm{bD} \\
(9.4)\end{array}$ & $\begin{array}{c}199.00 \mathrm{aA} \\
(4.1)\end{array}$ & $\begin{array}{c}151.6 \mathrm{bA} \\
(1.5)\end{array}$ & $\begin{array}{c}103.5 \mathrm{aA} \\
(3.3)\end{array}$ & $\begin{array}{c}112.4 \mathrm{aA} \\
(6.7)\end{array}$ \\
\hline \multicolumn{18}{|c|}{ One-way and Two-way ANOVA main effects } \\
\hline \multirow{3}{*}{\multicolumn{2}{|c|}{$\begin{array}{c}\text { Tissues } \\
\text { Concentration } \\
\text { Tissues } x \\
\text { Concentration }\end{array}$}} & \multirow{2}{*}{\multicolumn{2}{|c|}{$\begin{array}{c}0.000 * \\
0.229\end{array}$}} & & & & & & & 0.0 & & & & 0.0 & & & \\
\hline & & & & & 5 * & & & & & 0.0 & & & & 0. & & & \\
\hline & & & & & & & & & & 0. & & & & 0. & & & \\
\hline
\end{tabular}

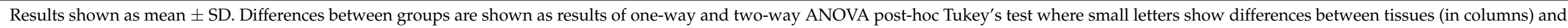
major letters show differences between concentrations (in rows). Means not showing the same letter are statically different. ${ }^{*}$ level of significant $(<0.05)$. 


\subsection{Profiling of HMs Related Gene Transcripts in Date Palm}

qPCR was performed for six genes; Pdpcs1, Pdmt3, Pdabcc family, Pdhma2, PdNramp6, and Pdmate5; in roots and leaves of date palm young plantlets grown during two months in $-\mathrm{Cd}$, or $-\mathrm{Cu}$, or $-\mathrm{Cr}$ spiked soils. The expression level of genes did not significantly differ between tissues of non-treated plants except for Pdpcs1 and Pdmate5, which were predominantly expressed in roots (Figure 2). However, metal contaminations strongly influenced the expression of all tested genes $(p<0.05$; Figure 2$)$ and differences were observed between tissues. For instance, the highest relative expression level (14.61) was observed for $P d m t 3$ in leaves exposed to high level of $\mathrm{Cr}$ (Figure 2b). Whereas, the highest expression factors of more than 80-, 110-, and 150-fold (data not shown) were obtained for Pdpcs1 in leaves of plants exposed to $50 \mathrm{mg} \mathrm{kg}^{-1} \mathrm{Cd}, 100 \mathrm{mg} \mathrm{kg}^{-1} \mathrm{Cu}$, and $500 \mathrm{mg} \mathrm{kg}^{-1}$ $\mathrm{Cr}$, respectively. Pdpcs1 transcript was mostly expressed in plantlets exposed at moderate concentration of $\mathrm{Cd}$, while it decreased at $100 \mathrm{mg} \mathrm{kg}^{-1} \mathrm{Cd}$. Gene downregulations were also observed for Pdmt3 and Pdmate5 in roots and leaves of plants exposed to high doses of $\mathrm{Cd}$ (Figure $2 \mathrm{~b}, \mathrm{f}$ ). Only $\mathrm{Cd}$ exposure caused downregulation of several genes. Downregulations were not observed in date palm exposed to $\mathrm{Cu}$ and $\mathrm{Cr}$. Pdhma2 was overexpressed in roots even at high $\mathrm{Cd}$ stress (Figure $2 \mathrm{~d}$ ). The latter gene was more induced by $\mathrm{Cr}^{\mathrm{VI}}$ ions. Its expression increased with increasing $\mathrm{Cu}$-stress in contrast to the other tested genes which expression decreased at $500 \mathrm{mg} \mathrm{kg}^{-1} \mathrm{Cu}$ (Figure 2). Gene expression patterns of MATE5 gene significantly varied between tissues (Figure 2f). Indeed, it was downregulated at high $\mathrm{Cd}$ concentration especially in leaves. However, Pdmate5 expression pattern was induced in Cr-treated leaves by almost 4-fold. The Nramp metal transporter gene expression has also been monitored (Figure 2e). In the present study, $\mathrm{Cd}^{2+}, \mathrm{Cu}^{2+}$, and $\mathrm{Cr}^{6+}$ ions induced PdNramp6 expression mainly in plant roots. Cr was the most effective PdNramp6 expression inductor.

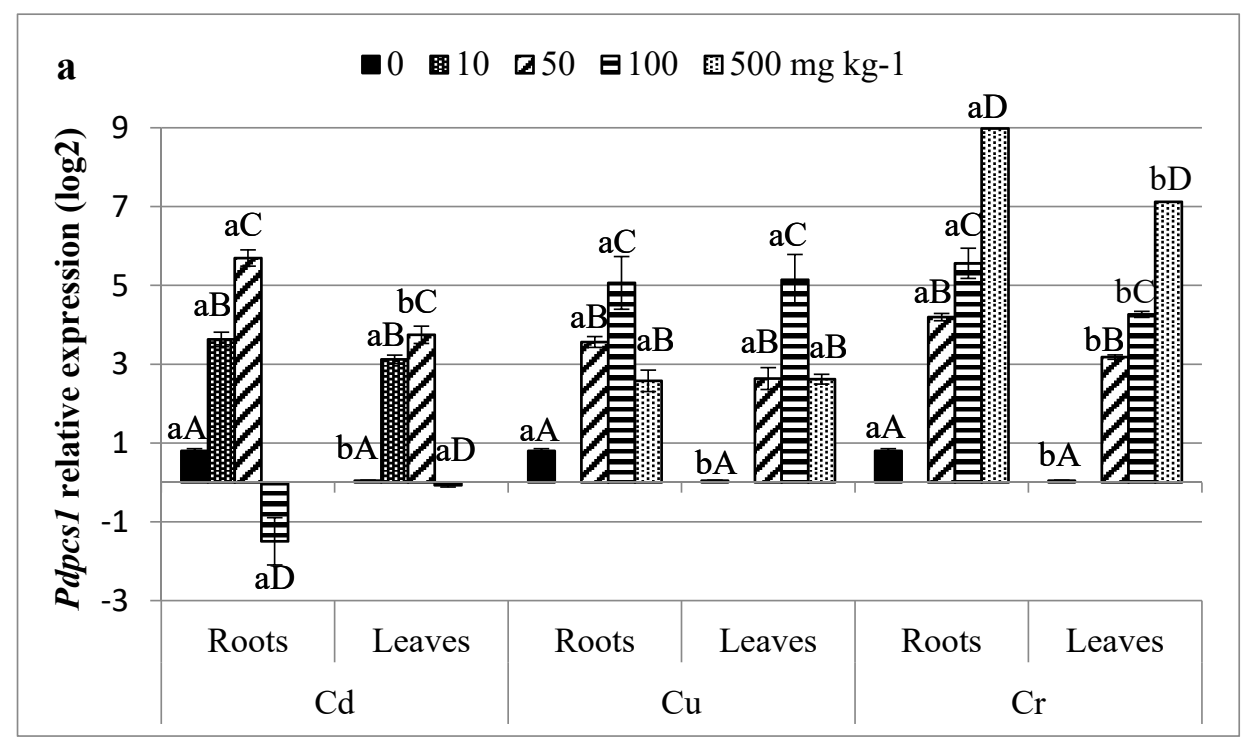

Figure 2. Cont. 

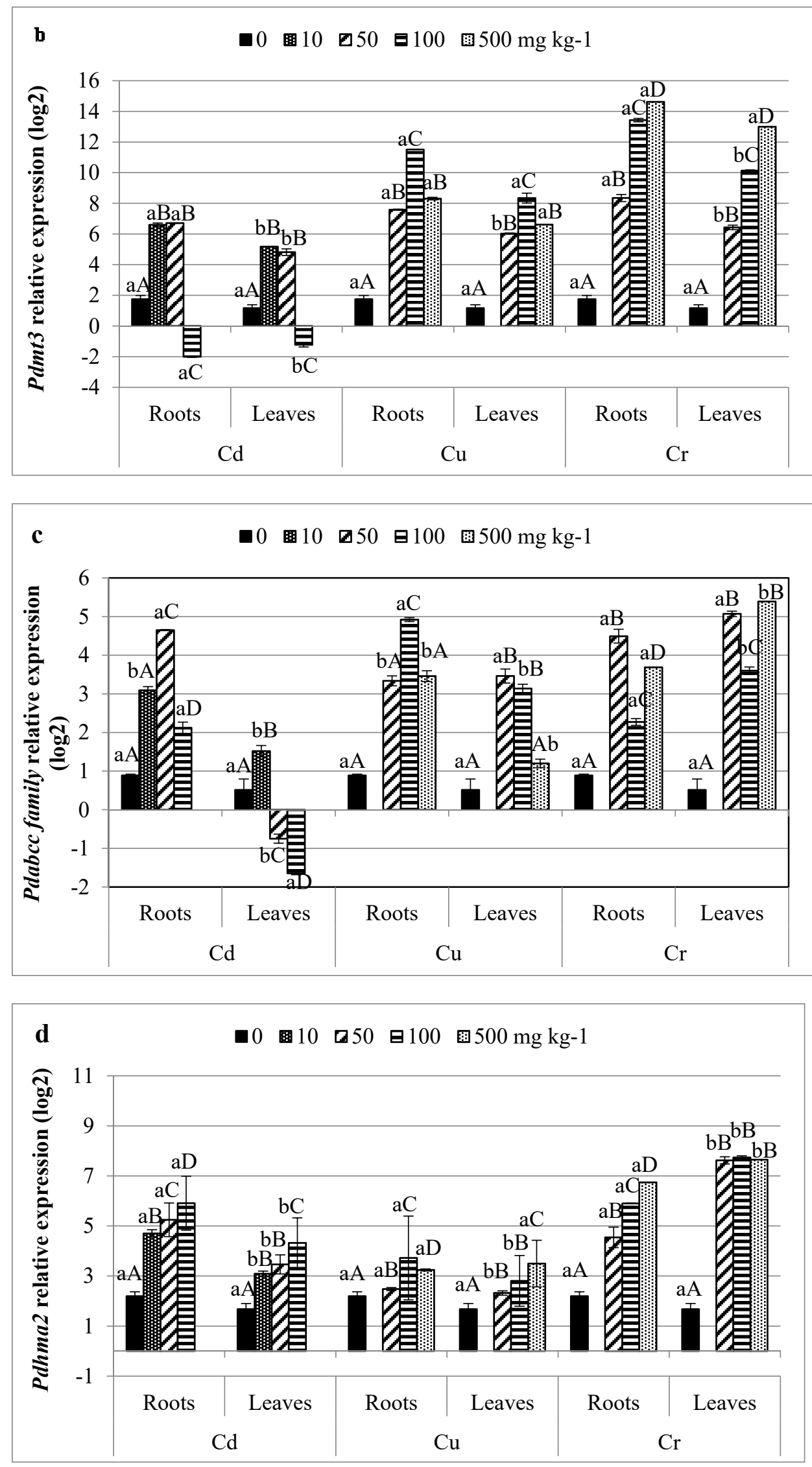

Figure 2. Cont. 

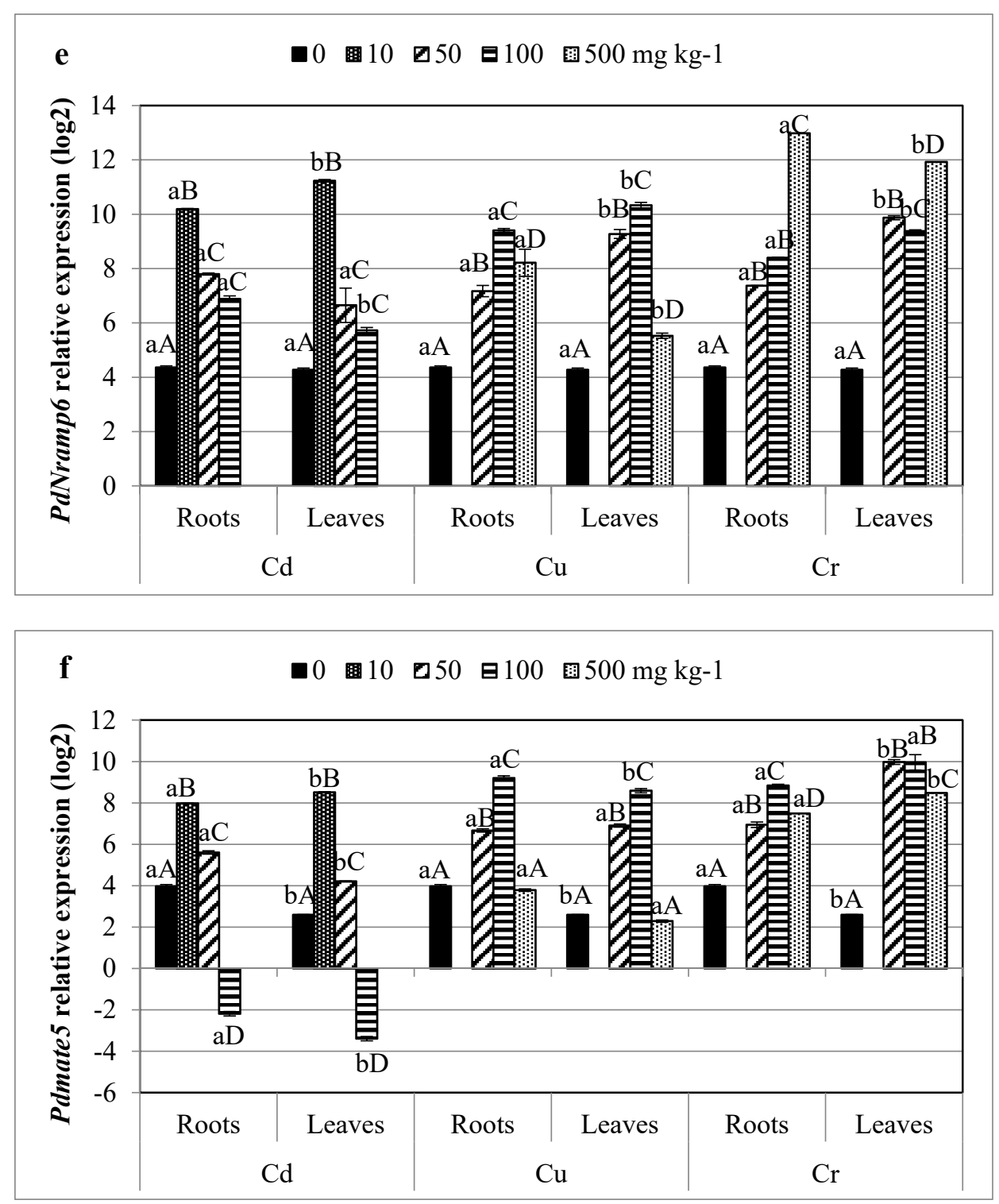

Figure 2. Relative expression factor of Pdpcs1 (a), Pdmt3 (b), Pdabcc (c), Pdhma2 (d), PdNramp6 (e), and Pdmate5 (f) exposed to $\mathrm{Cd}, \mathrm{Cu}$, and $\mathrm{Cr}$. Data presented are means \pm standard error of three independent experiments. Differences between groups are shown as results of one-way ANOVA post-hoc Tukey's test; where small letters show differences between tissues and major letters show differences between metal concentrations. Means not showing the same letter are statically different.

\section{Discussion}

\subsection{Metals Concentration and Their Interactions with Plant Nutrients}

Considered as the most industrialized city of Tunisia, the southern edge of Sfax city suffers for long time ago by emission of particles and uncontrolled waste storage, such as PG piles of ISPAF factory. It was found that the latter released around $4.5 \mathrm{t} /$ day of particulate matter containing sulfate, phosphorous compounds, and HMs in concentrations largely exceeding the permissible emission standards [53]. Arid climate of Sfax and especially low annual precipitations promotes the persistence of particles in the atmosphere. Metal-containing particles of the atmosphere may be directly retained by plant leaves or accumulate in soils after their deposition. To investigate date palm potential to uptake and transport metals, ex situ assays were performed in soil spiked with increasing concentrations of $\mathrm{Cd}, \mathrm{Cu}$ and $\mathrm{Cr}$. Results presented in Table 1 show great capacities of the plant to accumulate $\mathrm{Cd}$. Bioaccumulation factor of $\mathrm{Cd}$ ranged from 1.07 to 1.72 , suggesting metal transfer from soils treated with 10 and $50 \mathrm{mg} \mathrm{kg}^{-1}$ to the plant (Table 2). This ability 
to absorb metal may be related to its great TF against an important enrichment factor especially at low metal concentration (Table 2). Because metals may compete with each other, the abundance of a particular metal can disturb the uptake of others. For example, our results suggest that Cd can disturb Mn uptake by the plant (Table 3). Similarly, Dong et al. [54] indicated the existence of a negative correlation between $\mathrm{Cd}$ and Mn uptake in tomato plants exposed to Cd-stress. Such antagonism may restraint leaf photosynthesis and plant growth [55] observed in our case (data not shown). Non-essential metals may compete differently. For instance, $\mathrm{Cr}$ induced stress enhanced $\mathrm{Mn}$ accumulation in roots of the plant, while it decreased it in leaves (Table 3). In fact, previous reports showed that mobilization of micronutrients, especially $\mathrm{Mn}$, into the rhizosphere is due to its acidification and complexation with organic acids (citrate) in various plant species [56]. Yet, citric acid with its strong affinity to form complexes with $\mathrm{HM}$ reduces the mobility of metals to the shoots. Furthermore, the obtained values of BCF of $\mathrm{Cu}$ in roots of date palm at low metal amount (1.37), as well as those of TF (0.9), suggested that plants accumulate and stabilize this metal within these compartments. However, increasing $\mathrm{Cu}$ stress reduces metal absorption and translocation, especially at $500 \mathrm{mg} \mathrm{kg}^{-1} \mathrm{Cu}$, suggesting that date palm regulated intracellular $\mathrm{Cu}$ levels by regulating $\mathrm{Cu}$ absorption.

\subsection{Impact of Non-Essential Metal Uptake on Biochemical Integrative Biomarkers in Date Palm}

The ability of organisms to acclimate metabolically to metal stress in the environment has interested researchers for years [57]. In this context, $\mathrm{H}_{2} \mathrm{O}_{2}$ accumulation in plant is a key regulator in numerous physiological processes [58]. The risk of oxidative damage by accumulating $\mathrm{H}_{2} \mathrm{O}_{2}$, naturally exists in plant cells where oxidant accumulation are more important in green cells [59]. For instance, in control leaves, $\mathrm{H}_{2} \mathrm{O}_{2}$ level was 1.5 time more important than in control roots of date palm plantlets (Table 3). Therefore, with no ability to produce ROS directly, the mechanism of Cd-induced oxidative stress is different from other forms of stress [60]. Cd caused accumulation of some oxidant. Indeed, $\mathrm{H}_{2} \mathrm{O}_{2}$ amount in roots of young date palm plants grown in $100 \mathrm{mg} \mathrm{kg}^{-1}$ of $\mathrm{Cd}$ increased by more than 4 times compared to control (Table 4). This may be due to the fact that $\mathrm{Cd}$ decreases protective enzymatic defense mechanism, especially SOD activity. This latter decreased significantly with increasing Cd stress in roots and leaves parts of date palm (Table 4). Similarly, Romero-Puertas et al. [61] showed Cu/Zn SOD downregulation in pea plants exposed to $\mathrm{Cd}$ stress. Moreover, $\mathrm{Cd}$ may also displace Fe from proteins and increase free $\mathrm{Fe}$ that is responsible for ROS generation [60]. With a positive correlation between tissues and metal concentration $(p<0.05)$, APX activity increased in parallel to $\mathrm{H}_{2} \mathrm{O}_{2}$ accumulation in cells (Table 4). In fact, various environmental stimuli, such as $\mathrm{H}_{2} \mathrm{O}_{2}$, can modulate the expression of APX encoding genes [62]. Similarly, coffee cells increased APX activity at the lower Cd concentration [63]. At high metal amount, APX activity dropped down in roots. This may be due to glutathione (GSH) depletion and a subsequent reduction in the ascorbate-glutathione cycle [63]. In fact, the GSH reduction could be caused by PCs synthesis induction provoked by $\mathrm{Cd}^{2+}$ ions. Indeed, in the present research, Pdpcs1 gene encoding PCs synthesizer enzyme using GSH, showed the highest induction in roots treated with $50 \mathrm{mg} \mathrm{kg}^{-1} \mathrm{Cd}$ (Figure 2) were APX activity started to be reduced (Table 4). Furthermore, phenolic content of date palm cells was significantly affected by high Cd concentration (Table 4). With their antioxidant properties, phenolic compound accumulation in cells represents a key factor of induction of the defense mechanisms of plants through the phenylpropanoid pathway [64]. Significantly, higher accumulation of non-enzymatic oxidant scavengers was noted in leaves of date palm treated with $\mathrm{Cd}$ except for flavonoid content, which showed the highest induction in leaves of plants treated with $50 \mathrm{mg} \mathrm{kg}^{-1} \mathrm{Cd}$ (Table 4). It is oxidized by peroxidase, and it acts especially in $\mathrm{H}_{2} \mathrm{O}_{2}$-scavenging [65].

As a highly mobile strong oxidizer, $\mathrm{Cr}$ in its hexavalent form may cause severe phytotoxic effect. It enhanced $\mathrm{H}_{2} \mathrm{O}_{2}$ and TBARS level in cells (Table 3). $\mathrm{Cr}$ VI is catalytically more active than $\mathrm{Cd}$ and less active than $\mathrm{Cu}$, and is able to generate ROS via Fenton 
reaction [66]. As shown by Ahemad [66], $\mathrm{Cr}^{\mathrm{VI}}$ toxicity is related to its easy diffusion through the membrane cells, which leads to the production of free radicals. The relationship shown between $\mathrm{Cr}^{\mathrm{VI}}$ accumulation and oxidant generation can be understood as a negative influence of increasing metal accumulation on redox balance. The increase in $\mathrm{H}_{2} \mathrm{O}_{2}$ could be explained by the enhancement of SOD activity that is in correlation with the respective $\mathrm{Cr}$ accumulation in plant (Tables 1 and 3). In fact, Fe-SOD was found to be the predominant form of Cr-induced SOD in stressed plants [67]. However, excess of $\mathrm{Cr}$ was shown to interact with essential nutrient like Mn (Table 3) and especially Fe [68]. It decreased the uptake and translocation of Fe ions, decreasing SOD activity including Fe-SOD enzyme activity. APX continued to overproduce in all tested $\mathrm{Cr}$ concentrations and especially at the highest concentration (Table 4). This plant response to $\mathrm{Cr}$ defended the potential of APX to gain oxidative damage. Similar findings were reported in other plant species, such as sorghum $[67,69]$, suggesting that APX might have provided sufficient antioxidant defense against $\mathrm{H}_{2} \mathrm{O}_{2}$ generation. $\mathrm{Cr}$ salt also induced the biosynthesis of secondary metabolites especially polyphenol and flavonoids, particularly at $100 \mathrm{mg} \mathrm{kg}^{-1}$ of $\mathrm{Cr}$ (Table 4). Similar findings were shown in Brassica juncea exposed to $\mathrm{Cr}$ stress [70]. Moreover, Dubey et al. [71] reported that the most upregulated genes in response to Cr-stress are related to biosynthesis of secondary metabolites, especially flavonoid biosynthesis.

\subsection{Essential Metal Uptake Effects on Biochemical Integrative Biomarkers in Date Palm}

$\mathrm{Cu}$ is a transitional element and it is known to cause oxidative damage in cells [72]. Despite of its great accumulation in plant tissues, it was found that $\mathrm{Cu}$ was less active than $\mathrm{Cd}$ as a producer of oxidative stressor like $\mathrm{H}_{2} \mathrm{O}_{2}$. The fact that $\mathrm{Cu}^{2+}$ ions are used as cofactors by numerous proteins and are required by the ethylene receptor for proper signaling may explain in part this oxidative state of cells. Yet, SOD activity increased gradually, when metal concentration increased in the plant (Table 4). Cu ions are required in the chloroplast as cofactors for SOD producing $\mathrm{Cu} / \mathrm{Zn}$ SODs under a control of microRNAs [73,74]. Similarly to the behavior of Moso bamboo, (as Cu-hyperaccumulator plant) under cupric stress [75], the present results indicated that $P$. dactylifera can induce the activity of essential components of plant enzymatic antioxidant system, to avoid oxidative damage induced by $\mathrm{Cu}$ toxicity. However, high $\mathrm{Cu}$ stress caused overproduction of $\mathrm{H}_{2} \mathrm{O}_{2}$, decreased enzymes activity, and damaged biomolecules, such as membrane lipids (Table 4). Plants grown in soil containing $100 \mathrm{mg} \mathrm{kg}^{-1}$ of Cu secreted polyphenols, flavonoid, and tannins as additional mechanisms of self-protection to reduce toxic effect of $\mathrm{Cu}^{2+}$ (Table 4). Polyphenols are known to be involved in ROS scavenging due to their chemical structure which also helps to bind with HMs, especially Fe and $\mathrm{Cu}$ [76]. In addition, flavonoids are shown to be produced in oxidative stress conditions, where transition metals ions, such as $\mathrm{Fe}$ and $\mathrm{Cu}$, are involved. With their chelating properties and their ability to locate and neutralize ROS from cells, flavonoids have higher reducing capacity for $\mathrm{Cu}$ ions and act as ROS scavengers [38]. Similar increase in total flavonoids content was shown in the medicinal plant Orthosiphon stamineus exposed to Cu stress [77].

The biochemical responses described above, including integrative biomarkers for metallic stress, were further supplemented by the measure of the level of expression of selected genes.

\subsection{Infra-Individual Expression Biomarkers for HMs Stress on Date Palm}

Expression levels of six selected genes were monitored in date palm exposed to $\mathrm{Cd}$, $\mathrm{Cu}$, and $\mathrm{Cr}$ stress. Implication of metal chelators and transporters may be achieved by differential regulations at transcriptional and/or post-transcriptional levels. Only $\mathrm{Cd}$ at high concentration repressed expression of some genes (Figure 2), namely Pdpcs1, Pdabcc family, and Pdmate5. However, the highest Pdpcs1 induction was observed in leaves of plant treated with $50 \mathrm{mg} \mathrm{kg}^{-1} \mathrm{Cd}$ inducing the biosynthesis of PCS enzyme. Differently, Cobbett and Goldsbrough [22] have shown that in Arabidopsis PCS is constitutively expressed and post-translationally activated, especially by $\mathrm{Cd}^{2+}$. The synthesis of the small metal binding 
peptides (PCs) under control of PCS play a role in cellular homeostasis and trafficking of essential nutriment, particularly $\mathrm{Cu}$ and $\mathrm{Zn}[78,79]$. They are also required for detoxification of toxic HMs, such as Cd. In addition, increasing metal amount adversely affect $P d p c s$ expression in hypocotyls of date palms exposed to Cd, as shown previously [30]. In different plant tissues, increasing $\mathrm{Cr}$ concentration increased gene expression and the highest induction factor of Pdpcs 1 was observed at high concentration $\left(500 \mathrm{mg} \mathrm{kg}^{-1} \mathrm{Cr}\right.$ ), especially in date palm leaves (Figure 2a). PCs bind HM ions to form high-molecularweight (HMW) to be transported into the vacuoles across the tonoplast by the ABCC type transporters. A correlation between Pdpcs1 and Pdabcc-type expression was found (Figure 2a,c) and calculated by Statistica Pairwise Correlation coefficient $(r)$. It was found that at 10 and $50 \mathrm{mg} \mathrm{kg}^{-1} \mathrm{Cd}$, $r$ obtained values were almost equal to +1 ( 0.954 and 0.990 respectively), suggesting a perfect positive linear relationship. Furthermore, our results suggested that $\mathrm{Cu}$ and $\mathrm{Cr}$ ions were more likely to be up taken and translocated into vacuoles than $\mathrm{Cd}$ ions, which is in accordance to metal concentrations in plant tissues (Table 1). This may be a part of the plant defense mechanisms. Furthermore, Cr plant resistance may be explained by the overexpression of $m t 3$ (Figure $2 b$ ). In sorghum $\mathrm{Cr}^{\mathrm{VI}}$ tolerant variety, higher transcription rates of the MT3 were observed [69]. Others suggested that $\mathrm{H}_{2} \mathrm{O}_{2}$ produced under $\mathrm{Cr}$ stress acted as a signal to induce MT mRNA transcript. This is in accordance to our results which showed that $\mathrm{H}_{2} \mathrm{O}_{2}$ level in date palm treated with $\mathrm{Cr}$ and $\mathrm{Cu}$ increased with increasing metal amount (Table 3). Moreover, Pdmt3 was more responsive under $\mathrm{Cu}$ stress than under $\mathrm{Cd}$ stress suggesting that this gene could be specifically involved in Cu detoxification similarly to other species [80]. Furthermore, MTs participate in controlling the concentration of ROS that would activate defense mechanisms, e.g., via the mitogen-activated protein kinase (MAPK) cascade [81]. The complex HM-MT is then translocated into vacuole by ABCC transporters. However, the P-type ATPases proteins transport a variety of cations across cell membranes. The HM-transporting (HMA) subfamily of $\mathrm{P}_{1 \mathrm{~B}}$-ATPase contains different genes. HMAs involved in HMs uptake are known to be more selective than the other transporter proteins. HMAs gene belongs to the cation diffusion facilitator (CDF) protein family and is involved in $\mathrm{Zn}$ and $\mathrm{Cd}$ transport. Interest was focused on $h m a 2$, which respond differently to different metal stress (Figure 2d). In natural conditions, the expression of Pdhma2 was significantly $(p<0.05)$ similar in the roots and shoots accordingly to wheat plants [82]. It increased significantly with increasing metal stress, even at high metal amount. Pdhma2 relative expression factor reached 2.6 and 2.5 in roots, and leaves of $100 \mathrm{mg} \mathrm{kg}^{-1} \mathrm{Cd}$-treated plants, respectively. Studies in Arabidopsis showed the involvement of Athma2 and Athma4 in $\mathrm{Zn} / \mathrm{Cd}$ translocation through xylem loading, as they act as pumps to efflux $\mathrm{Zn} / \mathrm{Cd}$ out of cells suggesting a putative role of HMA2 in long-distance transportation. Yet, Pdhma2 relative expression factor was much higher under Cr conditions and it was induced even in $50 \mathrm{mg} \mathrm{kg}^{-1}$ of Cr. However, no significant effect of $\mathrm{Cr}$ concentrations on gene expression was shown in plant leaves compared to root tissues (Figure 2d). The gene maintained high expression level during metal presence in tissues. This finding suggested high hma2 sensitivity to $\mathrm{Cr}$ in date palm. In literature, no involvement of HMA2 in Cr uptake has been found yet. However, HMA5 encoding gene was upregulated by $\mathrm{Cr}^{\mathrm{VI}}$ ions and was indispensably implicated in the regulatory network responsive to $\mathrm{Cr}$ stress in radish [83]. Similarly, $\mathrm{Cr}$ induced Nramp6 expression by almost 3-fold in date palm roots and leaves (Figure 2e). The Nramp6 was more expressed in regulating Fe homeostasis and metal transport. The target gene expression enhancement under Cr-stress may be explained by the fact that the forms of $\mathrm{Cr}$ VI are reduced by $\mathrm{Fe}^{2+}$ uptake [81]. It was also induced in Cd stressed-plantlets, especially at low metal amount. Member 6 of Nramp family encoding gene was not much studied before. Yet, in rice, Nramp1 was found to be highly expressed in roots and shoots of plants treated with Cd contrarily to Nramp5 [84]. In addition, transporter encoding gene belongs to the family of citrate transporters (Multi antimicrobial extrusion protein MATE family protein); Pdmate5 expression responded to metal stress. MATE transporters are localized in both vacuole and plasma membranes, and are involved in a wide range of biological processes 
in plants, such as transport of secondary metabolites especially flavonoids, alkaloids, and anthocyanidins, detoxification of HMs, Fe translocation and Al detoxification, and efflux of plant hormones, such as abscisic acid [76]. mate are expressed especially in response to Fe ions presences in cells. Thereby, MATE proteins participate in Fe-citrate efflux and are engaged to translocate $\mathrm{Fe}^{2+}$ from the roots to leaves [85]. In our study, Pdmate5 transcription enhanced under stress except at high Cd level (Figure 2f). There is in correlation to flavonoids behavior under Cd stress. Similarly, MATE was found to be upregulated under $\mathrm{Cr}$-stress in rice as a transporter of flavonoids [71]. Because flavonoid composition is different in each plant species, the transport activity of MATE transporters could be different. However, in rice, MATE proteins were proved to be involved in $\mathrm{Cu}$ resistance by secreting $\mathrm{Cu}$-conjugates as secreting Al-citrate in response to Al-stress [86]. MATE were also upregulated under $\mathrm{Cu}$ stress in grapevine [87].

\section{Conclusions}

Facing to atmospheric emission by industries of Sfax region, the surrounding areas suffer from a metallic contamination. Focusing on the date palm as the perennial plant that was exposed to contamination for a long time, we demonstrated that $\mathrm{Cd}, \mathrm{Cu}$, and $\mathrm{Cr}$ levels in field collected plant leaves exceed the permissible limits. Nevertheless, plants continue to survive in such environment. The investigation for strategies for detoxification of HMs in the date palms, maintained in controlled conditions, made it possible to identify integrative biomarkers for each metal tested. Thus, non-specific biomarkers were monitored in plants exposed to $\mathrm{Cd}$ or $\mathrm{Cu}$ or $\mathrm{Cr}$ spiked soils. $\mathrm{H}_{2} \mathrm{O}_{2}$, TBARS, and CAT levels showed increase mainly in roots of Cd-exposed plantlets. However, SOD and APX activity and flavonoid production were stronger in response to excess $\mathrm{Cu}$ and $\mathrm{Cr}$. As specific biomarkers, gene regulatory networks play crucial roles in $\mathrm{Cu}$ homeostasis and $\mathrm{Cd}$ or $\mathrm{Cr}$ detoxification. $\mathrm{Cd}$ stress induced Pdpcs1 more than the Pdmt3 gene. Cd also increased the expression of the genes Pdhma2 and PdNramp6, which encoded proteins implied into translocation of metals to the leaves. This may explain the important TF of Cd in plantlets. Following exposure to $\mathrm{Cu}$, marked increases in the expression of Pdpcs1, Pdhma2 and PdNramp6 were noticed. Exposure to $\mathrm{Cr}$ induced Pdmt3 gene expression. Moreover, in the present study, we selected individual markers of HMs effects on date palm that include responses of the plant at a molecular level, and may also contribute to the design of genetic tools, to identify more efficient plants for phytoremediation.

Author Contributions: Conceptualization, F.V., A.E. and H.M.; Data curation, Z.C.; Formal analysis, Z.C. and A.R.; Funding acquisition, Z.C.; Investigation, Z.C.; Methodology, Z.C., W.K. and I.R.; Project administration, Z.C.; Software, Z.C. and I.R.; Supervision, F.V., H.M., F.V. and A.E.; Validation, Z.C. and A.R.; Visualization, I.R. and A.E. All authors have read and agreed to the published version of the manuscript.

Funding: This research received no external funding.

Institutional Review Board Statement: Not applicable.

Informed Consent Statement: Not applicable.

Data Availability Statement: Not applicable.

Acknowledgments: This research was supported by grants from the Tunisian ministry of Higher Education and Scientific research.

Conflicts of Interest: The authors declare no conflict of interest.

\section{References}

1. Peng, F.; Yin, H.; Cao, S.L.; Lou, W.Y. Chapter 6: Enzyme Nanocarries. In Advances in Enzyme Technology Biomass, Biofuels, Biochemicals; Elsevier: Amsterdam, The Netherlands, 2019; pp. 153-168.

2. Flora, S. Structural, chemical and biological aspects of antioxidants for strategies against metal and mettaloid exposure. Oxid Med. Cell Longev. 2009, 2, 191-206. [CrossRef] [PubMed] 
3. Bielen, A.; Remans, T.; Vangronsveld, J.; Cuypers, A. The influence of metal stress on the availability and redox state of ascorbate, and possible interference with its cellular functions. Int. J. Mol. Sci. 2013, 14, 6382-6413. [CrossRef] [PubMed]

4. Maresca, V.; Lettieri, G.; Sorbo, S.; Piscopo, M.; Basile, A. Biological Responses to Cadmium Stress in Liverwort Conocephalum conicum (Marchantiales). Int. J. Mol. Sci. 2020, 21, 6485. [CrossRef] [PubMed]

5. Toxicol, J.E.A.; Bansal, S.L.; Asthana, S. Environmental \& Analytical Toxicology Biologically Essential and Non-Essential Elements Causing Toxicity in Environment. J. Environ. Anal. Toxicol. 2018, 8. [CrossRef]

6. Guo, S.; Li, Q.; Ou-Yang, C.; Chen, L.; Wang, S.; Chen, F. Lead toxicity induced antioxidant enzyme and phenylalanine ammonia lyase activities in Jatropha curcas L. radicles. Fresenius Environ. Bull. 2009, 5, 811-815.

7. Festa, R.A.; Thiele, D.J. Magasine; Elsevier: Amsterdam, The Netherlands, 2011; pp. R877-R883.

8. Aghaz, M.; Bandehagh, A. Effect of lead stress on some leaf characteristics in dill (Anethom graveolens) ecotypes. Int. J. Agron. Plant Prod. 2013, 4, 147-150.

9. Palevsky, H.I.; Ribalet, F.; Swalwell, J.E.; Cosca, C.E.; Cokelet, E.D.; Feely, R.A.; Armbrust, E.V.; Quay, P.D. The influence of net community production and phytoplankton community structure on $\mathrm{CO}_{2}$ uptake in the Gulf of Alaska. Glob. Biogeochem. Cycles 2013, 27, 664-676. [CrossRef]

10. Ali, H.; Khan, E.; Ilahi, I. Environmental chemistry and ecotoxicology of hazardous heavy metals: Environmental persistence, toxicity, and bioaccumulation. J. Chem. 2019, 2019. [CrossRef]

11. Ismael, M.A.; Elyamine, A.M.; Moussa, M.G.; Cai, M.; Zhao, X.; Hu, C. Cadmium in plants: Uptake, toxicity, and its interactions with selenium fertilizers. Metallomics 2019, 11, 255-277. [CrossRef]

12. Zhang, X.; Zhang, D.; Sun, W.; Wang, T. The adaptive mechanism of plants to iron deficiency via iron uptake, transport, and homeostasis. Int. J. Mol. Sci. 2019, 20, 2424. [CrossRef]

13. Farhat, N.; Elkhouni, A.; Zorrig, W.; Smaoui, A.; Abdelly, C.; Rabhi, M. Effects of magnesium deficiency on photosynthesis and carbohydrate partitioning. Acta Physiol. Plant. 2016, 38. [CrossRef]

14. Wang, Y.X.; Hu, Y.; Zhu, Y.F.; Baloch, A.W.; Jia, X.M.; Guo, A.X. Transcriptional and physiological analyses of short-term Iron deficiency response in apple seedlings provide insight into the regulation involved in photosynthesis. BMC Genom. 2018, 19, 1-12. [CrossRef]

15. Xie, Y.; Ye, S.; Wang, Y.; Xu, L.; Zhu, X.; Yang, J. Transcriptome-based gene profiling provides novel insights into the characteristics of radish root response to $\mathrm{Cr}$ stress with next-generation sequencing. Front. Plant Sci. 2015, 6, 1-12. [CrossRef] [PubMed]

16. Sihag, S.; Brar, B.; Jashi, U. Salicylic acid induced amelioration of chromium toxicity and affects antioxydant enzyme activity in Sorghum biocolo L. Int. J. Phytoremediat. 2019, 21, 293-304. [CrossRef]

17. Singh, S.; Parihar, P.; Singh, R.; Singh, V.P.; Prasad, S.M. Heavy Metal Tolerance in Plants: Role of Transcriptomics, Proteomics, Metabolomics, and Ionomics. Front. Plant Sci. 2016, 6, 1-36. [CrossRef] [PubMed]

18. Boyd, R.S. Elemental Defenses of Plants by Metals. Nat. Educ. Knowl. 2010, 3, 57.

19. Rekik, I.; Chaâbene, Z.; Kriaa, W.; Rorat, A.; Franck, V.; Hafedh, M.; Elleuch, A. Transcriptome assembly and abiotic related gene expression analysis of date palm reveal candidate genes involved in response to cadmium stress. Comp. Biochem. Physiol. Part C 2019, 225, 108569. [CrossRef] [PubMed]

20. Dalcorso, G.; Farinati, S.; Furini, A. Regulatory networks of cadmium stress in plants. Plant Signal. Behav. 2010, 5, 663-667. [CrossRef]

21. Cobbett, C.S. Phytochelatin biosynthesis and function in heavy-metal detoxification. Curr. Opin. Plant Biol. 2000, 3, 211-216. [CrossRef]

22. Cobbett, C.; Goldsbrough, P. Phytochelatins and metallothineins: Roles in Heavy Metal Detoxification and Homeostasis. Annu. Rev. Plant Biol. 2002, 53, 159-182. [CrossRef]

23. Hassinen, V.; Vallinkoski, V.; Issakainen, S.; Tervahauta, A.; Ka, S.; Servomaa, K. Correlation of foliar MT2b expression with Cd and $\mathrm{Zn}$ concentrations in hybrid aspen (Populus tremula $\times$ tremuloides) grown in contaminated soil. Environ. Pollut. 2009, 157, 922-930. [CrossRef] [PubMed]

24. Klein, M.; Burla, B.; Martinoia, E. The multidrug resistance-associated protein (MRP/ABCC) subfamily of ATP-binding cassette transporters in plants. FEBS Lett. 2006, 580, 1112-1122. [CrossRef] [PubMed]

25. Kretzschmar, T.; Burla, B.; Lee, Y.; Martinoia, E.; Nagy, R. Functions of ABC transporters in plants. Essays Biochem. 2011, 50, 145-160. [CrossRef] [PubMed]

26. Palmer, C.; Guerinot, M. Lou A question of balance: Facing the challenges of Cu, Fe and Zn homeostasis. Nat. Chem. Biol. 2009, 5, 333-340. [CrossRef]

27. Burkhead, J.L.; Gogolin Reynolds, K.A.; Abdel-Ghany, S.E.; Cohu, C.M.; Pilon, M. Copper homeostasis. New Phytol. 2009, 182, 799-816. [CrossRef]

28. Lanquar, V.; Lelièvre, F.; Bolte, S.; Hamès, C.; Alcon, C.; Neumann, D.; Vansuyt, G.; Curie, C.; Schröder, A.; Krämer, U.; et al. Mobilization of vacuolar iron by AtNRAMP3 and AtNRAMP4 is essential for seed germination on low iron. EMBO J. 2005, 24, 4041-4051. [CrossRef]

29. Al-Busaidi, A.; Al-Yahyai, R.; Al-Said, F.; Ahmed, M. Tracking Heavy Metal Concentrations in Soils and Date Palms. Pak. J. Agric. Sci. 2015, 52, 129-134. 
30. Chaâbene, Z.; Rorat, A.; Rekik Hakim, I.; Bernard, F.; Douglas, G.C.; Elleuch, A.; Vandenbulcke, F.; Mejdoub, H. Insight into the expression variation of metal-responsive genes in the seedling of date palm (Phoenix dactylifera). Chemosphere 2018, 197, 123-134. [CrossRef]

31. Galfati, I.; Bilal, E.; Sassi, A.B.; Abdallah, H.; Zaier, A. Accumulation of heavy metals in native plants growing near the phosphate treatment industry, Tunisia. Carpathian J. Earth Environ. Sci. 2011, 6, 85-100.

32. Wali, A.; Colinet, G.; Khadhraoui, M.; Ksibi, M. Trace Metals in Surface Soil Contaminated by Release of Phosphate Industry in the Surroundings of Sfax-Tunisia. Environ. Res. Eng. Manag. 2013, 65, 20-30. [CrossRef]

33. Béjaoui, I.; Hadj, M.B.E.L. Cadmium contamination of local soils and vegetal in a tunisian phosphate plant environment. J. Nerw Sci. 2016, 26, 1445-1453.

34. Azri, C.; Maalej, A.; Tlili, A.; Medhioub, K. Characterization of the atmospheric pollution level in Sfax City (Tunisia): Influence of sources and meteorological factors. Tech. Sci. Méthodes Génie Urbain Génie Rural 2002, 1, 78-92.

35. Yanqun, Z.; Yuan, L.; Jianjun, C.; Haiyan, C.; Li, Q.; Schvartz, C. Hyperaccumulation of Pb, Zn and Cd in herbaceous grown on lead-zinc mining area in Yunnan, China. Environ. Int. 2005, 31, 755-762. [CrossRef] [PubMed]

36. Bu-Olayan, A.H.; Thomas, B.V. Environmental Sciences. Res. J. Environ. Sci. 2009, 3, 581-587. [CrossRef]

37. Elleuch, A.; Chaâbene, Z.; Grubb, D.C.; Drira, N.; Mejdoub, H.; Khemakhem, B. Morphological and biochemical behavior of fenugreek (Trigonella foenum-graecum) under copper stress. Ecotoxicol. Environ. Saf. 2013, 98, 46-53. [CrossRef] [PubMed]

38. Rustérucci, C.; Stallaert, V.; Milat, M.; Pugin, A.; Ricci, P.; Blein, J. Relationship between active oxygen species, lipid peroxidation, necrosis, and Phytoalexin Production lnduced by Elicitins in Nicofiana. Plant Physiol. 1996, 111, 885-891. [CrossRef]

39. Gómez-Vidal, S.; Tena, M.; Vicente Lopez-Llorca, L.; Jesús, S. Protein extraction from Phoenix dactylifera L. leaves, a recalcitrant material, for two-dimensional electrophoresis. Electrophoresis 2008, 29, 448-456. [CrossRef]

40. Bradford, M.M. A Rapid and Sensitive Method for the Quantitation Microgram Quantities of Protein Utilizing the Principle of Protein-Dye Binding. Anal. Biochem. 1976, 254, 248-254. [CrossRef]

41. Zayneb, C.; Imen, R.H.; Walid, K.; Grubb, C.D.; Bassem, K.; Franck, V.; Hafedh, M.; Amine, E. The phytochelatin synthase gene in date palm (Phoenix dactylifera L.): Phylogeny, evolution and expression. Ecotoxicol. Environ. Saf. 2017, 140, 7-17. [CrossRef]

42. Beyer, W.F.; Fridovich, I. Assaying for Superoxide Dismutase Activity: Some Large Consequences of Minor Changes in Conditions. Anal. Biochem. 1987, 161, 559-566. [CrossRef]

43. Aebi, H. Catalase in vitro. Methods Enzymol. 1984, 105, 121-126.

44. Nakano, Y.; Asada, K. Hydrogen Peroxide is Scavenged by Ascorbate-specific Peroxidase in Spinach Chloroplasts. Plant Cell Physiol. 1981, 22, 867-880.

45. Pinelo, M.; Rubilar, M.; Sineiro, J.; Nu, M.J. Extraction of antioxidant phenolics from almond hulls (Prunus amygdalus) and pine sawdust (Pinus pinaster). Food Chem. 2004, 85, 267-273. [CrossRef]

46. Zhishen, J.; Mengcheng, T.; Jianming, W. The determination of flavonoid contents in mulberry and their scavenging effects on superoxide radicals. Food Chem. 1999, 64, 555-559. [CrossRef]

47. Price, M.L.; Van Scoyoc, S.; Butler, L.G. A Critical Evaluation of the Vanillin Reaction as an Assay for Tannin in Sorghum Grain. J. Agric. Food Chem. 1978, 26, 1214-1218. [CrossRef]

48. Brulle, F.; Bernard, F.; Vandenbulcke, F.; Cuny, D.; Dumez, S. Identification of suitable qPCR reference genes in leaves of Brassica oleracea under abiotic stresses. Ecotoxicology 2014, 23, 459-471. [CrossRef]

49. Brulle, F.; Mitta, G.; Cocquerelle, C.; Vieau, D.; Lemiere, S.; Lepretre, A.; Vandenbulcke, F. Cloning and Real-Time PCR Testing of 14 Potential Biomarkers in Eisenia fetida Following Cadmium Exposure. Environ. Sci. Technol. 2006, 40, 2844-2850. [CrossRef]

50. Vodyanitskii, Y.N. Standards for the contents of heavy metals in soils of some states. Ann. Agrar. Sci. 2016, 14, 257-263. [CrossRef]

51. Kassir, L.N.; Lartiges, B.; Ouaini, N. Effects of fertilizer industry emissions on local soil contamination: A case study of a phosphate plant on the east Mediterranean coast. Environ. Technol. 2012, 33, 873-885. [CrossRef]

52. WHO. Permissible Limits of Heavy Metals in Soil and Plants; World Health Organization: Geneva, Switzerland, 1996.

53. Bahloul, M.; Chabbi, I.; Dammak, R.; Amdouni, R.; Medhioub, K.; Azri, C. Geochemical behaviour of PM10 aerosol constituents under the influence of succeeding anticyclonic/cyclonic situations: Case of Sfax City, southern Tunisia. Environ. Monit. Assess. 2015, 187, 757. [CrossRef]

54. Dong, J.; Wu, F.; Zhang, G. Influence of cadmium on antioxidant capacity and four microelement concentrations in tomato seedlings (Lycopersicon esculentum). Chemosphere 2006, 64, 1659-1666. [CrossRef] [PubMed]

55. Nazar, R.; Iqbal, N.; Masood, A.; Khan, M.I.R.; Syeed, S.; Khan, N.A. Cadmium Toxicity in Plants and Role of Mineral Nutrients in Its Alleviation. Am. J. Plant Sci. 2012, 03, 1476-1489. [CrossRef]

56. Millaleo, R.; Reyes-Diaz, M.; Ivanov, A.; Mora, M.; Alberdi, M. Manganese As Essential and Toxic Element for Plants: Transport, Accumulation and Resistance Mechanisms. J. Soil Sci. Plant Nutr. 2010, 10, 470-481. [CrossRef]

57. Tangahu, B.V.; Rozaimah, S.; Abdullah, S.; Basri, H.; Idris, M.; Anuar, N.; Mukhlisin, M. A Review on Heavy Metals (As, Pb, and $\mathrm{Hg}$ ) Uptake by Plants through Phytoremediation. Int. J. Chem. Eng. 2011, 31. [CrossRef]

58. Bright, J.; Desikan, R.; Hancock, J.T.; Weir, I.S.; Neill, S.J.; Lane, C.; Sciences, M.; Lane, C. ABA-induced NO generation and stomatal closure in Arabidopsis are dependent on $\mathrm{H}_{2} \mathrm{O}_{2}$ synthesis. Plant J. 2006, 45, 113-122. [CrossRef] [PubMed]

59. Gill, S.S.; Tuteja, N. Reactive oxygen species and antioxidant machinery in abiotic stress tolerance in crop plants. Plant Physiol. Biochem. 2010, 48, 909-930. [CrossRef] 
60. Hasanuzzaman, M.; Nahar, K.; Gill, S.S.; Alharby, H.F.; Razafindrabe, B.H.N.; Fujita, M. Hydrogen Peroxide Pretreatment Mitigates Cadmium-Induced Oxidative Stress in Brassica napus L.: An Intrinsic Study on Antioxidant Defense and Glyoxalase Systems. Front. Plant Sci. 2017, 8, 1-10. [CrossRef]

61. Romero-Puertas, M.C.; Palma, J.M.; Gómez, M.; Del Río, L.A.; Sandalio, L.M. Cadmium causes the oxidative modification of proteins in pea plants. Plant Cell Environ. 2002, 25, 677-686. [CrossRef]

62. Rosa, S.B.; Caverzan, A.; Teixeira, F.K.; Lazzarotto, F.; Silveira, J.A.G.; Ferreira-Silva, S.L.; Abreu-Neto, J.; Margis, R.; MargisPinheiro, M. Cytosolic APx knockdown indicates an ambiguous redox responses in rice. Phytochemistry 2010, 71, 548-558. [CrossRef]

63. Gomes-Junior, R.A.; Moldes, C.A.; Delite, F.S.; Pompeu, G.B.; Gratão, P.L.; Mazzafera, P.; Lea, P.J.; Azevedo, R.A. Antioxidant metabolism of coffee cell suspension cultures in response to cadmium. Chemosphere 2006, 65, 1330-1337. [CrossRef]

64. Ramakrishna, A.; Ravishankar, G.A. Influence of abiotic stress signals on secondary metabolites in plants. Plant Signal. Behav. 2011, 6, 1720-1731. [CrossRef]

65. Sharma, P.; Jha, A.B.; Dubey, R.S.; Pessarakli, M. Reactive Oxygen Species, Oxidative Damage, and Antioxidative Defense Mechanism in Plants under Stressful Conditions. J. Bot. 2012, 2012, 1-26. [CrossRef]

66. Ahemad, M. Enhancing phytoremediation of chromium-stressed soils through plant-growth-promoting bacteria. J. Genet. Eng. Biotechnol. 2015, 13, 51-58. [CrossRef]

67. Chatterjee, J.; Kumar, P.; Nand, P. Chromium toxicity induces oxidative stress in turnip. Indian J. Plant Physiol. 2015, 6, 15-163. [CrossRef]

68. Dube, B.K.; Tewari, K.; Chatterjee, J.; Chatterjee, C. Excess chromium alters uptake and translocation of certain nutrients in citrullus. Chemosphere 2003, 53, 1147-1153. [CrossRef]

69. Shanker, A.K.; Djanaguiraman, M.; Sudhagar, R.; Jayaram, K.; Pathmanabhan, G. Expression of metallothionein 3-like protein mRNA in sorghum cultivars under chromium (VI) stress. Curr. Sci. 2004, 86, 901-902.

70. Singh, S.; Sinha, S. Accumulation of metals and its effects in Brassica juncea (L.) Czern. (cv. Rohini) grown on various amendments of tannery waste. Ecotoxicol. Environ. Saf. 2005, 62, 118-127. [CrossRef]

71. Dubey, S.; Misra, P.; Dwivedi, S.; Chatterjee, S.; Bag, S.K.; Mantri, S.; Asif, M.H.; Rai, A.; Kumar, S.; Shri, M.; et al. Transcriptomic and metabolomic shifts in rice roots in response to $\mathrm{Cr}$ (VI) stress. BMC Genom. 2010, 11, 648. [CrossRef]

72. Cho, A.K.; Sioutas, C.; Miguel, A.H.; Kumagai, Y.; Schmitz, D.A.; Singh, M.; Eiguren-Fernandez, A.; Froines, J.R. Redox activity of airborne particulate matter at different sites in the Los Angeles Basin. Environ. Res. 2005, 99, 40-47. [CrossRef]

73. Abdel-Ghany, S.E.; Pilon, M. MicroRNA-mediated systemic down-regulation of copper protein expression in response to low copper availability in Arabidopsis. J. Biol. Chem. 2008, 283, 15932-15945. [CrossRef]

74. Gielen, H.; Remans, T.; Vangronsveld, J.; Cuypers, A. Toxicity responses of $\mathrm{Cu}$ and Cd: The involvement of miRNAs and the transcription factor SPL7. BMC Plant Biol. 2016, 16, 1-16. [CrossRef]

75. Chen, L.; Liu, Y.; Liu, H.; Kang, L.; Geng, J.; Gai, Y.; Ding, Y.; Sun, H.; Li, Y. Identification and expression analysis of MATE genes involved in flavonoid transport in blueberry plants. PLOS ONE 2015, 10, 1-20. [CrossRef] [PubMed]

76. Yadav, P.; Kaur, R.; Kohli, S.K.; Sirhindi, G.; Bhardwaj, R. Castasterone assisted accumulation of polyphenols and antioxidant to increase tolerance of B. juncea plants towards copper toxicity. Cogent Food Agric. 2016, 2, 1-14. [CrossRef]

77. Mamat, D.D.; Chong, C.S.; Samad, A.A.; Chai, T.T.; Manan, F.A. Effects of copper on total phenolics, flavonoids and mitochondrial properties of Orthosiphon stamineus callus culture. Int. J. Agric. Biol. 2015, 17, 1243-1248. [CrossRef]

78. Di Toppi, S.L.; Lambardi, M.; Pecchioni, N.; Pazzagli, L.; Durante, M.; Gabbrielli, R. Effects of Cadmium Stress on Hairy Roots of Daucus carota. J. Plant Physiol. 1999, 154, 385-391. [CrossRef]

79. Degola, F.; De Benedictis, M.; Petraglia, A.; Massimi, A.; Fattorini, L.; Sorbo, S.; Basile, A.; Sanita, L. A Cd/Fe/Zn-Responsive Phytochelatin Synthase is Constitutively Present in the Ancient Liverwort Lunularia cruciata (L.) Dumort. Plant Cell Physiol. 2014, 55, 1884-1891. [CrossRef]

80. Ramesh, G.; Podila, G.K.; Gay, G.; Marmeisse, R.; Reddy, M.S. Different patterns of regulation for the copper and cadmium Metallothioneins of the ectomycorrhizal fungus Hebeloma cylindrosporum. Appl. Environ. Microbiol. 2009, 75, $2266-2274$. [CrossRef]

81. Finatto, T.; de Oliveira, A.C.; Chaparro, C.; da Maia, L.C.; Farias, D.R.; Woyann, L.G.; Mistura, C.C.; Soares-Bresolin, A.P.; Llauro, C.; Panaud, O.; et al. Abiotic stress and genome dynamics: Specific genes and transposable elements response to iron excess in rice. Rice 2015, 8. [CrossRef]

82. Tan, J.; Wang, J.; Chai, T.; Zhang, Y.; Feng, S.; Li, Y.; Zhao, H.; Liu, H. Functional analyses of TaHMA2, a P 1B -type ATPase in wheat. Plant Biotechnol. J. 2013, 11, 420-431. [CrossRef]

83. Liu, W.; Xu, L.; Wang, Y.; Shen, H.; Zhu, X.; Zhang, K.; Chen, Y.; Yu, R.; Limera, C.; Liu, L. Transcriptome-wide analysis of chromium-stress responsive microRNAs to explore miRNA-mediated regulatory networks in radish (Raphanus sativus L.). Sci. Rep. 2015, 5, 1-17. [CrossRef]

84. Ishimaru, Y.; Takahashi, R.; Bashir, K.; Shimo, H.; Senoura, T.; Sugimoto, K.; Ono, K.; Yano, M.; Ishikawa, S.; Arao, T.; et al. Characterizing the role of rice NRAMP5 in Manganese, Iron and Cadmium Transport. Sci. Rep. 2012, 2, 1-8. [CrossRef] [PubMed]

85. Durrett, T.P.; Gassmann, W.; Rogers, E.E. The FRD3-Mediated Efflux of Citrate into the Root Vasculature Is Necessary for Efficient Iron Translocation. Plant Physiol. 2007, 144, 197-205. [CrossRef] [PubMed] 
86. Ritter, A.; Dittami, S.M.; Goulitquer, S.; Correa, J.A.; Boyen, C.; Potin, P.; Tonon, T. Transcriptomic and metabolomic analysis of copper stress acclimation in Ectocarpus siliculosus highlights signaling and tolerance mechanisms in brown algae. BMC Plant Biol. 2014, 14, 116. [CrossRef] [PubMed]

87. Leng, X.; Jia, H.; Sun, X.; Shangguan, L.; Mu, Q.; Wang, B.; Fang, J. Comparative transcriptome analysis of grapevine in response to copper stress. Sci. Rep. 2015, 5, 1-17. [CrossRef] [PubMed] 